\title{
Signal Synthesis in the Presence of an Inconsistent Set of Constraints
}

\author{
MARC GOLDBURG, MEMBER, IEEE AND ROBERT J. MARKS II, SENIOR MEMBER, IEEE
}

\begin{abstract}
In this paper, we present a novel technique for signal synthesis in the presence of an inconsistent set of constraints. This technique represents a general, minimum norm, solution to the class of synthesis problems in which: the desired signal may be characterized as being an element of some Hilbert Space; each of the $\boldsymbol{N}$ design constraints generates a closed convex set in that space; and those $N$ convex sets generate, or may be resolved into, two disjoint closed convex sets, such that at least one of the two sets is bounded. The synthesis technique employs alternating nearest point maps onto closed convex subsets of a Hilbert Space, and may be viewed as an extension of $D$. Youla's "Method of Convex Projections"- which addresses the case in which the $N$ closed convex sets, corresponding to the design constraints, possess a nonempty intersection. Section I provides a general introduction to the synthesis problem and to its solution. Section II contains the mathematical justification for the solution technique, while Section III presents an example of the synthesis of a data window for spectral estimation. In Section IV, we discuss potential extensions of this technique within the area of signal synthesis, as well as to the more general class of constrained optimization problems.
\end{abstract}

\section{INTRODUCTION}

W ITH THE increasing complexity of modern information transmission and processing systems, there have been concomitant increases in the variety, and in the stringency, of design constraints imposed upon the signals which these systems will utilize. It may well be the case that the set of constraints corresponding to the ideal signal for a particular application is inconsistent, in the sense that the satisfaction of any one of the constraints will preclude the satisfaction of other constraints in the set. Fxamples of such inconsistent constraint sets are abundant, and may be found in even the most fundamental of information systems.

Consider a simple baseband digital communications system, in which a single binary value is to be transmitted every $T$ seconds. In the $(k+1)$ th bit interval, the presence of a " 1 " at the source is to be indicated by the transmission of a pulse $p(t-k T)$-where $p(t)$ is identically zero for $t \notin[0, T]$ 一 while the presence of a " 0 " is to be indicated by the transmission of $-p(t-k T)$. Hence, $p(t)$ will be of infinite bandwidth, since any band-limited signal is analytic and thus cannot be zero over any continguous segment of the real line-unless it is identically zero for all values of its argument [1]. The channel over which the

Manuscript received May 16, 1984.

M. Goldburg was with the Department of Electrical Engineering, University of Washington, Seattle, WA. He is now with MIT Lincoln Laboratories, Lexington, MA 02173.

R. J. Marks II, is with the Department of Electrical Engineering, University of Washington, Seattle, WA 98195. pulse train travels will be band limited, due to physical laws, to some frequency $\Omega_{c}$. The filtering effect of the channel will thus cause a distorted version of the pulse train to be received, in which each transmitted pulse has spread into the following bit intervals; and the task of detection of the original binary sequence at the receiver is greatly complicated. The design constraints for the ideal $p(t)$-nonzero only for $t \in[0, T]$ and band limited to $\Omega_{c}$ - form an inconsistent set.

The same dichotomy of time and frequency constraints arises in the field of spectral estimation, where the ideal data window would be of finite duration in time and of vanishingly small bandwidth. A similar problem arises in two dimensions, as in the case of spectroscopy, where signals which are of finite extent in both the spatial and frequency planes would be desirable. In radar and other remote sensing systems, there may well be design constraints imposed upon the magnitude, phase, and spectral content of sensing signals, in such a manner as to form an inconsistent constraint set.

The objective of this paper is the presentation of an iterative signal synthesis technique, of general applicability in the presence of an inconsistent constraint set, which yields a signal most closely satisfying the various constraints. In order to make the meaning of the preceding statement more precise, the mathematical setting of the synthesis technique must be described. It is assumed that any desirable signal may be characterized as being an element of a Hilbert Space $-L_{2}(-\infty, \infty)$ and $l_{2}(-\infty, \infty)$, the spaces of all finite-energy continuous-time signals and discrete-time signals, respectively, are representative examples when equipped with the usual, respective, norms and inner products. It is further assumed that each constraint may be phrased in such a manner as to generate a closed convex set in that space. A subset of a linear space is said to be convex if, for any two elements $x_{1}$ and $x_{2}$ of that subset, the element $\lambda x_{1}+(1-\lambda) x_{2}$ is also in the subset for each value of $\lambda$ in the interval $(0,1)$. Subspaces, linear varieties (translates of subspaces), and the linear space itself are all subsumed under the category of convex sets, as well as many other subsets of the space which are meaningful in a signal synthesis context. In $L_{2}(-\infty, \infty)$ : the set of all signals bandlimited to some frequency $\Omega$ is closed and convex - a subspace; the set of all signals taking on prescribed values in some interval $\left[t_{1}, t_{2}\right]$ is closed and convex - a linear variety; the set of all signals whose energy does not exceed some fixed bound is closed and convex-a 
sphere centered at the origin; and the set of all signals satisfying a given dynamic range constraint is closed and convex. This listing is by no means exhaustive.

The final general criterion which the inconsistent constraint set must satisfy, in order that the synthesis technique of this paper be applicable, is that the constraint set must be resolvable into two disjoint. closed convex sets. There are two types of constraint sets which will meet this criterion. The first type is that in which the constraint set consists of only two constraints, each generating a closed convex set in the space, such that the two sets are disjoint. The example of the ideal design constraints for a baseband digital communications system, presented earlier in this section, is representative of this first type of constraint set. The second type of constraint set which will satisfy this criterion consists of $N>2$ constraints each generating a closed convex set $C_{i}, i=1, \cdots, N$, such that the set $\left\{C_{1}, C_{2}, \cdots, C_{N}\right\}$ may be represented by the two sets $\left\{C_{k_{1}}, C_{k_{2}}, \cdots, C_{k_{N_{1}}}\right\}$ and $\left\{C_{l_{1}}, C_{l_{2}}, \cdots, C_{l_{N_{2}}}\right\}$, where
(a)

$$
\begin{array}{ll}
C_{k_{i}} \in\left\{C_{1}, \cdots, C_{N}\right\}, & \text { for } i=1, \cdots, N_{1} \\
C_{l_{i}} \in\left\{C_{1}, \cdots, C_{N}\right\}, & \text { for } i=1, \cdots, N_{2}
\end{array}
$$$$
C_{l_{i}} \in\left\{C_{1}, \cdots, C_{N}\right\}
$$$$
\left\{\bigcap_{i=1}^{N_{1}} C_{k_{i}}\right\} \cap C_{j} \neq\{\varnothing\} \quad \text { or }\left\{\bigcap_{i=1}^{N_{2}} C_{l_{i}}\right\} \cap C_{j} \neq \varnothing,
$$
(c) $\left\{\bigcap_{i=1}^{N_{1}} C_{k_{i}}\right\} \cap\left\{\bigcap_{i=1}^{N_{2}} C_{l_{i}}\right\}=\{\varnothing\}$.
for each $j=1, \cdots, N$

Since the intersection of closed convex sets is itself closed and convex, the two disjoint sets $\left\{\bigcap_{i=1}^{N_{1}} C_{k_{i}}\right\}$ and $\left\{\bigcap_{i=1}^{N_{2}} C_{l_{1}}\right\}$ are each closed and convex. Furthermore, some portion of each constraint set in $\left\{C_{1}, \cdots, C_{N}\right\}$ lies in at least one of the intersections. If two sets $\left\{C_{k_{1}}, \cdots, C_{k_{N_{1}}}\right\}$ and $\left\{C_{l_{1}}, \cdots, C_{l_{N}}\right\}$ can be constructed from the elements of $\left\{C_{1}, \cdots, C_{N}\right\}$ such that conditions (a), (b) and (c) above are satisfied, then $\left\{\bigcap_{i=1}^{N_{1}} C_{k_{i}}\right\}$ and $\left\{\bigcap_{i=1}^{N_{2}} C_{l_{i}}\right\}$ will be a resolution of $\left\{C_{1}, \cdots, C_{N}\right\}$ into two disjoint closed convex sets in the sense of the criterion. All following discussion of the synthesis technique of this paper will be phrased in terms of two disjoint closed convex sets, with the understanding that these two sets may have been generated by the resolution of a constraint set with more than two elements, in the manner just described.

A rigorous mathematical discussion of the iterative synthesis technique of this paper will be presented in Section II. A qualitative discussion is, however, in order at this point. It may be shown that to each closed convex set $C$ in a Hilbert Space $H$, there corresponds an operator, whose domain is $H$ and whose range is $C$, which is the nearest point map from $H$ into $C$. Applied to any $x \in H$, this operator will yield the unique element of $C$ closest, in the sense of the norm, to $x$. The more familiar class of orthogonal projection operators corresponding to subspaces of $H$ is, in fact, subsumed by the category of nearest point maps into closed convex subsets of $H$.

Consider the case of two disjoint closed convex sets $C_{1}$ and $C_{2}$ with respective nearest point maps $P_{1}$ and $P_{2}$; and form the composite operator $P=P_{1} \circ P_{2}$ (i.e., $P x=$

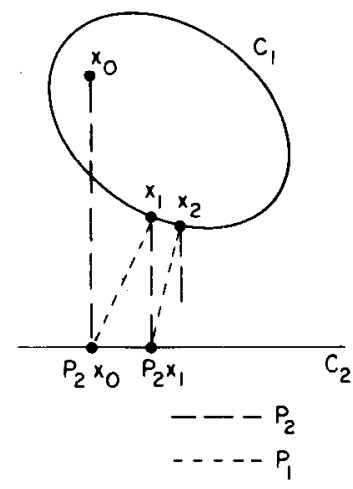

Fig. 1. Alternating nearest point maps between disjoint convext sets.

$P_{1}\left(P_{2} x\right)$ for $\left.x \in H\right)$. Let $x_{0}$ be some element of $C_{1}$, and let $x_{1}=P x_{0} . P_{1}$ and $P_{2}$ are nearest point maps, so it must be the casc that $\left\|x_{1}-P_{2} x_{0}\right\| \leq\left\|x_{0}-P_{2} x_{0}\right\|-x_{1}$ can be no farther from $C_{2}$ than $x_{0}$. Similarly, if $x_{2}=P x_{1}=P^{2} x_{0}$, it will be true that

$$
\left\|x_{2}-P_{2} x_{1}\right\| \leq\left\|x_{1}-P_{2} x_{1}\right\| \leq\left\|x_{1}-P_{2} x_{0}\right\| \leq\left\|x_{0}-P_{2} x_{0}\right\| .
$$

The relationship between $x_{0}, x_{1}$, and $x_{2}$ is displayed in Fig. 1.

Proceeding on a purely intuitive basis, one might suppose that the sequence of iterates $\left\{P^{n} x_{0}\right\}_{n=1}^{\infty}$ will converge, or, since $P x \in C_{1}$ for all $x \in H$, that the sequence $\left\{P^{n}(P x)\right\}_{n=1}^{\infty}$ will converge for an arbitrary element of $x$ of $H$. If the sequence indeed converges, it will converge, by definition, to a fixed point of $P$. In order for some $u \in C_{1}$ to be fixed point of $P, u$ must be $P u=P_{1}\left(P_{2} u\right)-P_{2} u$ is the closest element of $C_{2}$ to $u \in C_{1}$, and $u$ is the element of $C_{1}$ closest to $P_{2} u \in C_{2}$. If $u$ is a fixed point of $P$, then $\left\|u-P_{2} u\right\|$ will clearly be a local minimum for the distance between elements of $C_{1}$ and elements of $C_{2}$.

Section II is primarily concerned with the proof of two statements. Firstly, that if $u \in C_{1}$ is a fixed point of $P$, then $\left\|u-P_{2} u\right\|$ is a global minimum for the distance between elements of $C_{1}$ and elements of $C_{2}-$ thus $u$ and $P_{2} u$ are signals most closely satisfying the inconsistent constraints represented by $C_{1}$ and $C_{2}$. Secondly, that with a simple modification of the operator $P$, denoted by $P_{\lambda}$, a form of convergence to a fixed point of $P$ can be guaranteed for the sequence of iterates $\left\{P_{\lambda}^{n}(P x)\right\}_{n=1}^{\infty}$ for any $x \in H$.

Historically, distinctions have not been made between iterative signal synthesis algorithms and iterative signal restoration algorithms in a Hilbert Space setting [2]. In the presence of a consistent constraint set, the difference between a synthesis problem and a restoration problem is merely one of the particular constraints which must be satisfied. In the former case the constraints represent the desired properties of the resultant signal, while in the latter they represent a priori knowledge of the characteristics of the signal to be restored. In light of the inconsistency of the constraint set which the technique of this paper employs, this technique will be primarily applicable to problems of synthesis. However, it does have two important antecedents in the class of iterative synthesis and restoration techniques. 
The first of these is the so-called Superresolution or Error Energy Reduction algorithm-initially presented by Papoulis [3]-[4] and Gerchberg [5], and later generalized by a number of researchers [6]-[9]. The value of the Gerchberg-Papoulis technique is that it provides a practical algorithm for the reconstruction of a bandlimited signal solely from knowledge of its values over some interval, whereas a formal mathematical technique such as analytic continuation is primarily of pedagogical worth.

The second antecedent of the synthesis technique of this paper is D. Youla's "Method of Convex Projections" [10]. Youla's description of this technique was the first systematic presentation of signal synthesis and restoration phrased in terms of closed convex sets in a Hilbert Space. The Method of Convex Projections is applicable to situations in which a consistent set of $N$ constraints generates $N$ closed convex sets, whose intersection is nonempty. Denoting the nearest point maps corresponding to the $N$ convex sets $C_{1}, \cdots, C_{N}$ as $P_{1}, \cdots, P_{N}$, respectively, Youla guarantees a form of convergence for the sequence $\left\{\left(P_{1} \circ P_{2} \circ \cdots \circ P_{N}\right)^{n} x\right\}_{n=1}^{\infty}$ to an element of $\left\{\bigcap_{k=1}^{N} C_{k}\right\}$, for any element of $x$ of the respective Hilbert space [10].

One of the most valuable aspects of the paper describing the Method of Convex Projections is the inclusion of a compilation of nearest point maps, for a number of closed convex sets, which are of frequent interest within the context of signal synthesis and restoration [10]. While the orthogonal projection operator corresponding to a particular subspace may often be ascertained by inspection, the nearest point map corresponding to some more general type of convex set may not, in general, be so simply determined. The generation of the nearest point map for a particular convex set will be mentioned again in Sections II and IV.

Section I has, hopefully, provided the reader with some intuition for signal synthesis in a Hilbert Space setting, and with an awareness for the synthesis techniques which have been available to date. Although there are some important mathematical differences between the setting for Youla's "Method of Convex Projections" and that of signal synthesis in the presence of an inconsistent constraint set, the latter should be thought of as the natural conceptual extention of the former. In Section II, we will provide a rigorous mathematical discussion of signal synthesis in the presence of an inconsistent set of constraints.

\section{THEORY}

\subsection{Introduction}

As in Section I, $P$ will be defined to be the composition $P_{1} \circ P_{2}$, where $P_{1}$ and $P_{2}$ are the nearest point maps corresponding to the two disjoint closed convex sets $C_{1}$ and $C_{2}$ in a Hilbert Space $H$. A detailed study will be undertaken of the fixed points of the operator $P$ and of the convergence properties of the sequence

$$
\left\{(\lambda I+(1-\lambda) P)^{n}(P x)\right\}_{n=1}^{\infty}=\left\{P_{\lambda}^{n}(P x)\right\}_{n=1}^{\infty},
$$

for arbitrary $x \in H$.
Most of the important results presented here were culled from articles which appeared in the mathematics literature, and the accompanying proofs were often terse. In the interest of an intuitively satisfying, complete presentation, an effort has been made to minimize the number of assertions stated without proof.

Section II is divided into eight parts. Section 2.2 discusses the idea of uniformly convex Banach Spaces, of which Hilbert Spaces are a subset. Section 2.3 deals with the properties of the nearest point maps $P_{1}$ and $P_{2}$, and with the composition operator $P$; and Section 2.4 describes the properties of the set of fixed points of $P$. Sections 2.5 and 2.6 treat the notions of asymptotic regularity and weak convergence, respectively - both of which will be necessary for the convergence proof of Section 2.7. Section 2.8 is a synopsis.

\subsection{Uniformly Convex Spaces}

The concept of a uniformly convex Banach Space is central to the following development. The notion was first introduced by Clarkson [11] in 1936 and it has been advantageously employed in a variety of contexts. The usual definition is as follows [12]: A Banach Space $X$ is said to be uniformly convex if, for all $\epsilon>0$, there exists a $\delta=\delta(\epsilon)>0$ such that $\|x\| \leq 1,\|y\| \leq 1$ and $\|x-y\|>\epsilon$, taken together, imply that $\frac{1}{2}\|x+y\| \leq(1-\delta)$, where $x$ and $y$ are elements of $X$. An immediate consequence is that the boundary of the unit ball in such a space contains no line segments. All Hilbert Spaces are uniformly convex-this may be observed in the "parallelogram law" which holds in such spaces,

$$
\|x+y\|^{2}+\|x-y\|^{2}=2\left(\|x\|^{2}+\|y\|^{2}\right) .
$$

The following Lemma describes some implications of uniform convexity.

Lemma 1: Let $X$ be a uniformly convex Banach Space. Let $x$ and $y$ be elements of $X$ such that $\|x\| \leq M,\|y\| \leq M$, - for some fixed $M>0$; and suppose that $\|x-y\| \geq \epsilon>0$. Then the following statements are true.

A) There exists a $\delta=\delta(\epsilon)>0$ such that

$$
\frac{1}{2}\|x+y\| \leq(1-\delta) \max \{\|x\|,\|y\|\} .
$$

B) For all $\alpha \in(0,1)$, there exists a $\delta=\delta(\epsilon, \alpha)>0$ such that

$$
\|\alpha x+(1-\alpha) y\| \leq(1-\delta) \max \{\|x\|,\|y\|\} .
$$

Proof of $A$ :

Let $\rho=\max \{\|x\|,\|y\|\}$, and consider $\|x / \rho-y / \rho\| \geq$ $\epsilon / \rho>0$. Now, $\|x / \rho\| \leq 1$ and $\|y / \rho\| \leq 1$, and our previous definition of uniform convexity yields

$$
\frac{1}{2}\left\|\frac{x}{\rho}+\frac{y}{\rho}\right\| \leq(1-\delta), \quad \delta>0
$$

or

$$
\frac{1}{2}\|x+y\| \leq(1-\delta) \rho=(1-\delta) \max \{\|x\|,\|y\|\} .
$$

Thus statement $A$ is true.

Proof of $B$ :

Case 1, $\alpha=1 / 2$ : In this event, statement $B$ reduces to statement $A$ and is, therefore, valid. 
Case 2, $\alpha<1 / 2$ : We note that $\alpha x+(1-\alpha) y=y+$ $\alpha(x-y)=(1 / 2)(y)+(1 / 2)(y+2 \alpha(x-y))$, i.e., the midpoint of the line segment joining the point $y$ to the point $y+$ $2 \alpha(x-y)$. Since $\|x-y\| \geq \epsilon$, then $\|y-(y+2 \alpha(x-y))\|$ $=\|2 \alpha(x-y)\| \geq 2 \alpha \epsilon>0$. Applying statement A, there exists a $\delta>0$ such that

$$
\begin{aligned}
\|\alpha x+(1-\alpha) y\| & =(1 / 2)\|y+(y+2 \alpha(x-y))\| \\
& \leq(1-\delta) \max \{\|y\|,\|y+2 \alpha(x-y)\|\}
\end{aligned}
$$

Suppose $\|y\| \geq\|x\|$, then

$$
\begin{aligned}
\|y+2 \alpha(x-y)\| & \leq\|(1-2 \alpha) y\|+\|2 \alpha x\| \\
& \leq(1-2 \alpha)\|y\|+2 \alpha\|y\| \\
& =\|y\| .
\end{aligned}
$$

Similarly, if $\|x\| \geq\|y\|,\|y+2 \alpha(x-y)\| \leq\|x\|$. So, there exists a $\delta>0$ such that

$$
\|\alpha x+(1-\alpha) y\| \leq(1-\delta) \max \{\|y\|,\|x\|\}, \quad \alpha<1 / 2 \text {. }
$$

Case 3, $\alpha>1 / 2$ : Making the identification $\alpha x+(1-\alpha) y$ $=(1 / 2)(x-2(1-\alpha)(x-y))+(1 / 2)(x)$, and proceeding as in Case 2, we find that

$$
\begin{array}{r}
\|\alpha x+(1-\alpha) y\| \leq(1-\delta) \max \{\|y\|,\|x\|\}, \\
\alpha>1 / 2, \delta>0 .
\end{array}
$$

So statement $\mathbb{B}$ is true, and the proof of the lemma is complete.

In fact, statements $A$ and $B$ are each necessary and sufficient conditions for the uniform convexity of $X$. That this should be so is straightforward in the case of $A$, while the verification in the case of $B$ is more involved-the proof, in terms of sequences in $X$, appears in a paper by Helmut Schaefer [13]. Lemma 1 will be adequate in the present context.

One implication of uniform convexity, which will be invoked in the next lemma, is a theorem due to D. Milman which asserts that every uniformly convex Banach Space is reflexive [12]. The reflexive character of Hilbert Spaces may be ascertained without specific reference to uniform convexity, but in the case of more general Banach Spaces, this result, known as Milman's Theorem, provides a definitive test for the reflexive property.

\subsection{Nearest Point Maps in Hilbert Spaces}

With the concept of a uniformly convex Space at our disposal, an examination of nearest point maps and compositions of nearest point maps may be undertaken. Let $C_{1}$ be a closed convex set in a uniformly convex Banach Space, $X$. The nearest point map, $P_{1}$, corresponding to $C_{1}$ is formally defined for any $x \in X$ as follows [14]:

$$
\left\|x-P_{1} x\right\|=\inf _{y \in C_{1}}\|x-y\| .
$$

The following lemma addresses the questions of existence and uniqueness for nearest point maps.

Lemma 2: Let $C_{1}$ be a closed convex set in a normed linear space, $E$.

A) A corresponding nearest point map, $P_{1}$, exists if $E$ is reflexive.
B) If $P_{1}$ exists and $E$ is strictly convex, then $P_{1}$ is unique in the sense that $P_{1} x \in C_{1}$ is unique for any $x \in E$. A normed linear space is said to be strictly convex if, for any two elements $x$ and $y$ such that $\|x-y\| \neq 0$ and $\|x\|=\|y\|=1,\|\lambda x+(1-\lambda) y\|<1$ for $\lambda \in(0,1)[15]$. Strict convexity is clearly subsumed by uniform convexity, as is the reflexive property by virtue of Milman's Theorem. The result is that if $C_{1}$ is a closed convex set in a uniformly convex Banach Space, then the associated nearest point map, $P_{1}$, exists and is unique (in the sense of B). The proof of statement $\mathrm{A}$ is beyond the scope of this discussion and the reader is referred to two papers by Phelps [15], [16]. The proof of statement $B$ relies upon the strict convexity of $E$, and will not be presented at this point, as a similar argument is made in the proof of forthcoming Theorem 3.

The observation should be made that Lemma 2 provides no insight into the functional form of the nearest point map and that the construction of such a mapping operator for a particular convex set is usually a nontrivial task. In practical situations, nearest point maps tend to be nonlinear-some examples in the Hilbert Spaces $L_{2}(-\infty, \infty)$ and $L_{2}(-\infty, \infty) \times L_{2}(-\infty, \infty)$ are given in a paper by Youla [10].

It is clear that in a uniformly convex Banach Space, $X$, the nearest point map $P_{1}$ corresponding to a closed convex set $C_{1}$ is a projection in the sense that $P_{1} x=P_{1}\left(P_{1} x\right)=P_{1}^{2} x$ for any $x \in X$. The terms projection and nearest point map will be used interchangeably from this point on.

The next lemma asserts that the projection operator corresponding to a closed convex set in a Hilbert space is nonexpansive. An operator $T$ in a normed linear space is said to be nonexpansive if, for any two elements of the space in the domain of $T$, say $w_{1}$ and $w_{2}$, the following statement is true:

$$
\left\|T w_{1}-T w_{2}\right\| \leq\left\|w_{1}-w_{2}\right\| .
$$

The nonexpansivity of such projection operators in a Hilbert Space setting is an important result, but the proof will require the invocation of the fact that if $P_{1}$ is the projection operator corresponding to a closed convex set $C_{1}$ in a Hilbert Space $H$, and if $x$ is any element of $H$, then $\operatorname{Re}\left(x-P_{1} x \mid y-P_{1} x\right) \leq 0$ for all $y$ in $C_{1}$-where $\operatorname{Re} \beta$ represents the real part of the, possibly complex, scalar $\beta$ [14].

The truth of this statement may be verified by noting that the line segment $\lambda y+(1-\lambda) P_{1} x, \lambda \in[0,1]$, lies in $C_{1}$ for all $y \in C_{1}$ and all $x \in H$. By the definition of $P_{1}$,

$$
\begin{aligned}
\left\|x-P_{1} x\right\|^{2} \leq & \left\|x-\left(\lambda y+(1-\lambda) P_{1} x\right)\right\|^{2} \\
= & \left\|\left(x-P_{1} x\right)+\lambda\left(P_{1} x-y\right)\right\|^{2} \\
= & \left\|x-P_{1} x\right\|^{2}+\lambda\left\{\left(x-P_{1} x \mid P_{1} x-y\right)^{*}\right. \\
& \left.+\left(P_{1} x-y \mid x-P_{1} x\right)^{*}\right\}+\lambda^{2}\left\|P_{1} x-y\right\|^{2} \\
= & \left\|x-P_{1} x\right\|^{2}+2 \lambda \operatorname{Re}\left(x-P_{1} x \mid P_{1} x-y\right) \\
& +\lambda^{2}\left\|P_{1} x-y\right\|^{2} .
\end{aligned}
$$

$$
2 \operatorname{Re}\left(x-P_{1} x \mid y-P_{1} x\right) \leq \lambda\left\|P_{1} x-y\right\|^{2} .
$$


Since this holds for all $\lambda \in[0,1]$, it must hold for $\lambda=0$, yielding the desired result:

$$
\operatorname{Re}\left(x-P_{1} x \mid y-P_{1} x\right) \leq 0, \quad x \in H, y \in C_{1} .
$$

Lemma 3: Let $C_{1}$ be a closed convex set in a Hilbert Space $H$ with associated projection operator $P_{1}$. Then $P_{1}$ is a nonexpansive operator with domain $H$ [10].

Proof: Let $x_{1}$ and $x_{2}$ be any two elements of $H$. By

$$
\operatorname{Re}\left(x_{1}-P_{1} x_{1} \mid P_{1} x_{2}-P_{1} x_{1}\right) \leq 0
$$

and

$$
\operatorname{Re}\left(x_{2}-P_{1} x_{2} \mid P_{1} x_{1}-P_{1} x_{2}\right) \leq 0
$$

So

$$
\begin{aligned}
0 \geq & \operatorname{Re}\left\{\left(x_{1}-P_{1} x_{1} \mid P_{1} x_{2}-P_{1} x_{1}\right)\right. \\
& \left.+\left(x_{2}-P_{1} x_{2} \mid P_{1} x_{1}-P_{1} x_{2}\right)\right\} \\
= & \operatorname{Re}\left\{\left(x_{1} \mid P_{1} x_{2}-P_{1} x_{1}\right)-\left(P_{1} x_{1} \mid P_{1} x_{2}-P_{1} x_{1}\right)\right. \\
& \left.+\left(x_{2} \mid P_{1} x_{1}-P_{1} x_{2}\right)-\left(P_{1} x_{2} \mid P_{1} x_{1}-P_{1} x_{2}\right)\right\} \\
= & \operatorname{Re}\left\{-\left(x_{1} \mid P_{1} x_{1}-P_{1} x_{2}\right)+\left(P_{1} x_{1} \mid P_{1} x_{1}-P_{1} x_{2}\right)\right. \\
& \left.+\left(x_{2} \mid P_{1} x_{1}-P_{1} x_{2}\right)-\left(P_{1} x_{2} \mid P_{1} x_{1}-P_{1} x_{2}\right)\right\} \\
= & \operatorname{Re}\left\{\left(P_{1} x_{1}-P_{1} x_{2} \mid P_{1} x_{1}-P_{1} x_{2}\right)\right. \\
& \left.-\left(x_{1}-x_{2} \mid P_{1} x_{1}-P_{1} x_{2}\right)\right\} .
\end{aligned}
$$

Thus

$$
\left\|P_{1} x_{1}-P_{1} x_{2}\right\|^{2}-\operatorname{Re}\left(x_{1}-x_{2} \mid P_{1} x_{1}-P_{1} x_{2}\right) \leq 0
$$

and

$$
\left|\left(x_{1}-x_{2} \mid P_{1} x_{1}-P_{1} x_{2}\right)\right| \geq \operatorname{Re}\left(x_{1}-x_{2} \mid P_{1} x_{1}-P_{1} x_{2}\right) .
$$

Therefore,

$$
\left\|P_{1} x_{1}-P_{1} x_{2}\right\|^{2} \leq\left|\left(x_{1}-x_{2} \mid P_{1} x_{1}-P_{1} x_{2}\right)\right| .
$$

Using the Cauchy-Schwarz inequality,

$$
\left\|P_{1} x_{1}-P_{1} x_{2}\right\|^{2} \leq\left\|x_{1}-x_{2}\right\| \cdot\left\|P_{1} x_{1}-P_{1} x_{2}\right\|
$$

whence $\left\|P_{1} x_{1}-P_{1} x_{2}\right\| \leq\left\|x_{1}-x_{2}\right\|$, completing the proof. The reader should note that no assumptions of linearity on the part of $P_{1}$ were required.

The next Theorem establishes the nonexpansive nature of the composite operator $P$ employed in the iterative synthesis procedure.

Theorem 1: Let $C_{1}$ and $C_{2}$ be two disjoint closed convex sets in a Hilbert Space $H$. Let $P_{1}$ and $P_{2}$ be the respective projection operators associated with these sets. Let $P$ be the composite operator defined by $P x=\left(P_{1} \circ P_{2}\right) x=$ $P_{1}\left(P_{2} x\right)$, for all $x \in H$. Then $P$ is nonexpansive.

Proof: Let $x_{1}$ and $x_{2}$ be arbitrary elements of $H . P_{1}$ and $P_{2}$ are nonexpansive, therefore,

$$
\begin{aligned}
\left\|P x_{1}-P x_{2}\right\| & =\left\|P_{1}\left(P_{2} x_{1}\right)-P_{1}\left(P_{2} x_{2}\right)\right\| \\
& \leq\left\|P_{2} x_{1}-P_{2} x_{2}\right\| \leq\left\|x_{1}-x_{2}\right\| .
\end{aligned}
$$

\subsection{Fixed Points}

This section is primarily concerned with the structure of the fixed point set of the composite operator $P$. The onus for almost all of the restrictions on the general applicability of the iterative signal synthesis technique must be ascribed to the results of this section, but, as will be seen, these restrictions imbue the fixed-point set of $P$ with some very desirable properties.

The following theorem provides a sufficient condition for the existence of a fixed-point set for $P$.

Theorem 2: Let $X$ be a uniformly convex Banach Space, $C$ a bounded, closed convex subset of $X$, and $T$ a nonexpansive mapping of $C$ into itself. Then $T$ has a fixed point in $C$ [17].

The proof of this theorem is beyond the scope of this discussion. What is salient, though, is the requirement that $C$ be closed and bounded. As regards the composite operator $P=P_{1} \circ P_{2}$, the restriction is that the convex set $C_{1}$, in which the fixed points of $P$ will lie, must be closed and bounded. The qualification that $C_{1}$ be bounded is, in a purely abstract setting, relatively severe. In a physical setting, however, there is always an ultimate power or energy constraint-corresponding closely, for instance, to a bound on the $L_{2}$ norm - upon a signal; and the limitation imposed by Theorem 2 upon $C_{1}$ is not a restriction in the sense of a physically realizable signal. The restrictions on $C_{2}$ remain those of closure and convexity, and $C_{2}$ may well be unbounded, as in the case of a subspace. In all following discussion, therefore, $C_{1}$ will be considered to be bounded as well as closed.

The following lemma, due to Helmut Schaefer [13], concerns the structure of the set of fixed points of a nonexpansive operator, and leads to Corollary 1 which will be used in the proof of the convergence properties of the sequence $\left\{P_{\lambda}^{n}(P x)\right\}$.

Lemma 4: Let $C$ be a bounded, closed convex set in a Hilbert Space $H$. Let $T$ be a nonexpansive mapping of $H$ into $C$, with fixed point set $F_{T}$ in $C$. Then $F_{T}$ is a convex subset of $C$.

Proof: Suppose that $F_{T}$ has at least two distinct elements, and let $u_{1}$ and $u_{2}$ be two such elements. Let $u_{0}$ be any element of the set

$$
\left\{x: x \in H, x=\lambda u_{1}+(1-\lambda) u_{2}, 0<\lambda<1\right\}
$$

the line segment joining $u_{1}$ and $u_{2}$; and define

$$
\begin{array}{ll}
Z_{1}^{\prime}=u_{0}-u_{1} & Z_{1}=T u_{0}-u_{1} \\
Z_{2}^{\prime}=u_{2}-u_{0} & Z_{2}=u_{2}-T u_{0} .
\end{array}
$$

Thus

$$
Z_{1}^{\prime}+Z_{2}^{\prime}=Z_{1}+Z_{2}
$$

Furthermore,

$$
\left\|Z_{1}\right\|=\left\|T u_{0}-u_{1}\right\|=\left\|T u_{0}-T u_{1}\right\| \leq\left\|u_{0}-u_{1}\right\|=\left\|Z_{1}^{\prime}\right\| .
$$

Similarly, $\left\|Z_{2}\right\| \leq\left\|Z_{2}^{\prime}\right\|$, yielding

$$
\left\|Z_{1}+Z_{2}\right\| \leq\left\|Z_{1}\right\|+\left\|Z_{2}\right\| \leq\left\|Z_{1}^{\prime}\right\|+\left\|Z_{2}^{\prime}\right\| .
$$

Substituting for $u_{0}$

$$
\begin{aligned}
\left\|Z_{1}^{\prime}\right\|+\left\|Z_{2}^{\prime}\right\| & =\left\|(1-\lambda)\left(u_{2}-u_{1}\right)\right\|+\left\|\lambda\left(u_{2}-u_{1}\right)\right\| \\
& =\left\|u_{2}-u_{1}\right\| \\
& =\left\|Z_{1}^{\prime}+Z_{2}^{\prime}\right\|=\left\|Z_{1}+Z_{2}\right\| .
\end{aligned}
$$


Combining this with (2),

$$
\left\|Z_{1}+Z_{2}\right\|=\left\|Z_{1}\right\|+\left\|Z_{2}\right\|=\left\|Z_{1}^{\prime}\right\|+\left\|Z_{2}^{\prime}\right\|=\left\|Z_{1}^{\prime}+Z_{2}^{\prime}\right\| .
$$

Since $\left\|Z_{i}\right\| \leq\left\|Z_{i}^{\prime}\right\|, i=1,2$, it must be the case that $\left\|Z_{i}\right\|=$ $\left\|Z_{i}^{\prime}\right\|, i=1,2$. For any two elements, $x_{1}$ and $x_{2}$, of $H$ it is true that

$$
\begin{aligned}
\left\|x_{1}+x_{2}\right\|^{2} & =\left\|x_{1}\right\|^{2}+2 \operatorname{Re}\left(x_{1} \mid x_{2}\right)+\left\|x_{2}\right\|^{2} \\
& \leq\left\|x_{1}\right\|^{2}+2\left|\left(x_{1} \mid x_{2}\right)\right|+\left\|x_{2}\right\|^{2} \\
& \leq\left\|x_{1}\right\|^{2}+2\left\|x_{1}\right\| \cdot\left\|x_{2}\right\|+\left\|x_{2}\right\|^{2}
\end{aligned}
$$

with equality if and only if $x_{2}=\gamma x_{1}$, where $\gamma$ is an element of the scalar field over which $H$ is defined.

By virtue of (3), then, $Z_{2}=\gamma Z_{1}$ and $Z_{2}^{\prime}=\gamma^{\prime} Z_{1}^{\prime}$, for some nonzero scalars $\gamma$ and $\gamma^{\prime}$. From the definitions of the $Z_{i}$ 's and $Z_{i}^{\prime}$ 's,

$$
Z_{1}+\gamma Z_{1}=Z_{1}^{\prime}+\gamma^{\prime} Z_{1}^{\prime}
$$

or

$$
Z_{1}=\frac{1+\gamma^{\prime}}{1+\gamma} Z_{1}^{\prime}
$$

But $\left\|Z_{1}\right\|=\left\|\left(\left(1+\gamma^{\prime}\right)\right) /(1+\gamma) Z_{1}^{\prime}\right\|=\left\|Z_{1}^{\prime}\right\| \neq 0$, therefore, $\gamma$ $=\gamma^{\prime}$ and $Z_{i}=Z_{i}^{\prime}, i=1,2$. It is now readily observable that $u_{0} \in F_{T}$ as

$$
Z_{1}-Z_{2}=2 T u_{0}-\left(u_{1}+u_{2}\right)=2 u_{0}-\left(u_{1}+u_{2}\right)=Z_{1}^{\prime}-Z_{2}^{\prime}
$$

which yields $T u_{0}=u_{0}$. Since a particular value of $\lambda$ was not specified in the definition of $u_{0}, F_{T}$ is proven to be both closed and convex.

The following corollary of Lemma 4 characterizes subsets of $F_{T}$ in terms of the limit point of the sequence $\left\{T^{n} x\right\}$, where $x$ is any element of $H$ and $T$ is the operator of Lemma 4. The corollary will be invoked in Theorem 5 , the convergence theorem for the sequence $\left\{P_{\lambda}^{n}(P x)\right\}$. By the nonexpansivity of $T$, the sequence $\left\{\left\|T^{n} x-u\right\|\right\}$ is monotone nonincreasing for any $u \in F_{T}$, since

$$
\left\|T^{n} x-u\right\|=\left\|T^{n} x-T u\right\| \leq\left\|T^{n-1} x-u\right\|, \quad n=1,2, \cdots .
$$

Thus for any $u \in F_{T}$, there exists the non negative limit

$$
d(u)=\lim _{n \rightarrow \infty}\left\|T^{n} x-u\right\|
$$

for each $x \in H$. Consider the following subset of $F_{T}$, for some fixed $x$ in $H$,

$$
F_{d}=\left\{u \in F_{T}: d(u) \leq d\right\}
$$

for some fixed nonnegative constant $d$.

Corollary 1: Let $T, C$ and $H$ be as in Lemma 4. Let $F_{d}$, a subset of $F_{T}$, be that of the preceding definition, where $x$ is any fixed element of $H$ [18].

A) For any $d \geq 0, F_{d}$ is a bounded, closed convex subset of $F_{T}$, nonempty if $d$ is large enough.

B) Let $\Delta$ be the smallest value of $d$ such that $F_{d}$ is nonempty; then the set $F_{\Delta}$ consists of a single element.

Proof of $A$ :

As $C$ is closed and bounded, $F_{T}$ will be nonempty by virtue of Theorem 2. $F_{T}$ and $F_{d}$, subsets of $C$, are clearly bounded. Suppose that $F_{d}$ consists of more than one element for some $d>0$, and let $u_{1}$ and $u_{2}$ be any two distinct elements thereof. Consider, for any $\alpha \in(0,1)$.

$$
\begin{aligned}
& \left\|T^{n} x-\left(\alpha u_{1}+(1-\alpha) u_{2}\right)\right\| \\
& \quad=\left\|\alpha\left(T^{n} x-u_{1}\right)+(1-\alpha)\left(T^{n} x-u_{2}\right)\right\| \\
& \quad \leq \alpha\left\|T^{n} x-u_{1}\right\|+(1-\alpha)\left\|T^{n} x-u_{2}\right\|, \quad \text { all } n .
\end{aligned}
$$

Therefore,

$$
\lim _{n \rightarrow \infty}\left\|T^{n} x-\left(\alpha u_{1}+(1-\alpha) u_{2}\right)\right\| \leq d .
$$

It was established in Lemma 4 that $F_{T}$ was convex, thus $\left(\alpha u_{1}+(1-\alpha) u_{2}\right) \in F_{T}$ and it is thus an element of $F_{d}$, by (4). Hence, $F_{d}$ is convex and closed; and statement $\mathrm{A}$ is verified.

Proof of $B$ :

Case $1, \Delta=0$ :

If $\Delta=0, u \in F_{\Delta}$ if and only if $\lim _{n \rightarrow \infty}\left\|T^{n} x-u\right\|=0$, i.e., $\left\{T^{n} x\right\}$ converges strongly to $u$. For a given $x \in H$, there can be only a single distinct element of $F_{T}$ satisfying this condition, and $F_{\Delta}=\{u\}$.

Case 2, $\Delta>0$ :

In this case, the proof is by contradiction. Suppose that $F_{\Delta}$ consists of more than one distinct element, and let $u_{1}$ and $u_{2}$ be any two such elements. Then $\left\|u_{1}-u_{2}\right\|>0$. By the uniform convexity of $H$, there exists a $\delta=\delta(\alpha)>0$ for all $\alpha \in(0,1)$ such that

$$
\begin{aligned}
& \left\|\alpha\left(T^{n} x-u_{1}\right)+(1-\alpha)\left(T^{n} x-u_{2}\right)\right\| \\
& \leq(1-\delta) \max \left\{\left\|T^{n} x-u_{1}\right\|,\left\|T^{n} x-u_{2}\right\|\right\},
\end{aligned}
$$

all $n$ since $u_{1}-u_{2}=\left(T^{n} x-u_{2}\right)-\left(T^{n} x-u_{1}\right)$. Hence,

$$
\left\|T^{n} x-\left(\alpha u_{1}+(1-\alpha) u_{2}\right)\right\| \leq(1-\delta) \Delta, \quad \text { as } n \rightarrow \infty \text {. }
$$

Thus $d\left(\alpha u_{1}+(1-\alpha) u_{2}\right)<\Delta, 0<\alpha<1$. $F_{\Delta}$ is convex, and so $\left(\alpha u_{1}+(1-\alpha) u_{2}\right) \in F_{\Delta}$, but the existence of some $u \in F_{\Delta}$ for which $d(u)<\Delta$ contradicts the definition of $F_{\Delta}$. Whence, $F_{\Delta}$ consists of a single element, and corollary is established.

The goal of the iterative signal synthesis technique, as stated in Section I, is to generate ordered pairs of points, each pair containing one element from $C_{1}$ and one from $C_{2}$, such that the distance between the elements of each pair is a minimum. The following theorem addresses this issue. One should note that the statement of the theorem assumes that the Hilbert Space $H$ is defined over the scalar field of real numbers. This is the second restriction imposed upon the synthesis technique in Section 2.4., as $H$, if it is interpreted as a function space, must then be composed entirely of real-valued functions or of imaginary-valued functions, but may not contain complex-valued functions. The synthesis technique may still be employed to generate complex-valued functions, however, if an appropriate product space $-H_{1} \times H_{2}$, where $H_{1}$ consists of real-valued functions and $\mathrm{H}_{2}$ consists of imaginary-valued functions-can be constructed with a real-valued inner product.

There are two additional concepts which will be required in the proof of the theorem. The first is that of the distance bctween the sets $C_{1}$ and $C_{2}$,

$$
d\left(C_{1}, C_{2}\right)=\min _{\substack{x_{1} \in C_{1} \\ x_{2} \in C_{2}}}\left\|x_{1}-x_{2}\right\|
$$




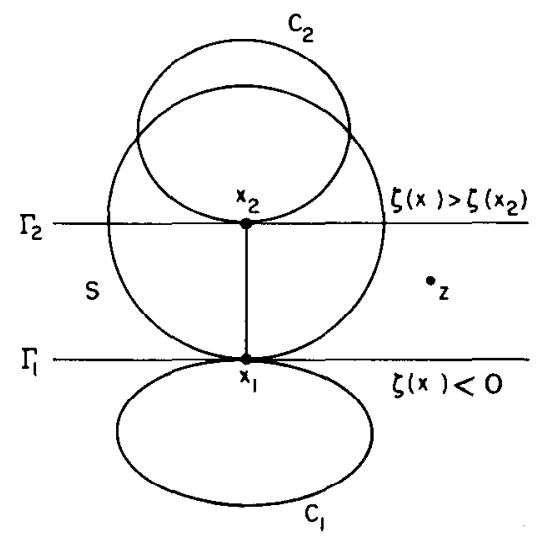

Fig. 2. Distance between the members of each element of $D_{p}$.

which is well defined since $C_{1}$ and $C_{2}$ are closed. $F_{P}$, the set of fixed points of the composite operator $P=P_{1} \circ P_{2}$, is a subset of $C_{1}$; and the set of dual fixed points of $P$ is defined as

$$
D_{P}=\left\{\left(x_{1}, x_{2}\right): x_{1} \in F_{P}, x_{2}=P_{2} x_{1}\right\} .
$$

In light of Lemma 2 , for each $\left(x_{1}, x_{2}\right) \in D_{P}, x_{2}$ is uniquely determined by $x_{1}$. Each element of $F_{P}$ corresponds to a single element-an ordered pair-of $D_{P}$.

Theorem 3: Let $H$ be a Hilbert Space defined over the scalar field of real numbers; and let $C_{1}$ and $C_{2}$ be two disjoint, closed convex sets in $H$. Furthermore, let $C_{1}$ be bounded. Define $P_{1}$ and $P_{2}$ to be the respective projection operators for $C_{1}$ and $C_{2}$, and define the composite operator $P=P_{1} \circ P_{2}$. Denote the set of fixed points of $P$ by $F_{P}$ and its set of dual fixed points by $D_{P}$. Then, for each $\left(x_{1}, x_{2}\right) \in D_{P}$,

$$
\left\|x_{1}-x_{2}\right\|=d\left(C_{1}, C_{2}\right) .
$$

Proof: By Theorem 2, $D_{P}$ and $F_{P}$ are not empty. Referring to Fig. 2, let $x_{1}$ be an element of $F_{P}$ and $\left(x_{1}, x_{2}\right)$ be the corresponding element of $D_{P} . x_{1}$ may be assumed to be the origin, $\phi$, with no loss of generality, since that assumption only implies a translation in $H$.

There exists a hyperplane $\Gamma_{1}=\{x \in H: \zeta(x)=0\}$ containing $x_{1}$, where $\zeta$ is a linear functional on $H$, such that $\left(x_{2}-x_{1}\right)$ is orthogonal to $\Gamma_{1}$ and $\zeta\left(x_{2}\right)>0$ [19]. Now, $C_{1}$ lies entirely in the closed halfspace $\{x \in H: \zeta(x) \leq 0\}$, a claim which will be substantiated through a proof by contradiction.

Suppose that there exists a $z \in C_{1}$ such that $\zeta(z)>0$, and consider the closed sphere

$$
\begin{aligned}
S & =\left\{x \in H:\left\|x_{2}-x\right\| \leq\left\|x_{2}-x_{1}\right\|\right\} \\
& =\left\{x \in H:\left\|x_{2}-x\right\| \leq\left\|x_{2}\right\|\right\}
\end{aligned}
$$

since $x_{1}=\phi . S$ is the collection of all points in $H$ which are no further from $x_{2}$ than $x_{1}$. Since $C_{1}$ is convex, it contains the segment $(1-\lambda) x_{1}+\lambda z=\lambda z$, for $0 \leq \lambda \leq 1$. It will be shown that a subset of this segment lies within $S$, violating the condition that $x_{2}=P_{2} x_{1}$.

If, in fact, some subset of that segment is contained in the interior of $S$, it will be the case that

$$
\left\|x_{2}-x_{1}\right\|>\left\|x_{2}-\left((1-\lambda) x_{1}+\lambda z\right)\right\| .
$$

Equivalently,

$$
\begin{aligned}
\left\|x_{2}\right\|^{2} & >\left\|x_{2}-\lambda z\right\|^{2} \\
& =\left\|x_{2}\right\|^{2}-2\left(x_{2} \mid \lambda z\right)+\|\lambda z\|^{2}
\end{aligned}
$$

or

$$
\begin{gathered}
2 \lambda\left(z \mid x_{2}\right)>\lambda^{2}\|z\|^{2} \\
\lambda<\frac{2\left(z \mid x_{2}\right)}{\|z\|^{2}} .
\end{gathered}
$$

Since $\|z\|^{2}>0$, values of $\lambda$ such that $(1-\lambda) x_{1}+\lambda z$ lays in the interior of $S$ will exist if $\left(z \mid x_{2}\right)>0$. Consider the projection of $z$ onto the subspace spanned by $x_{2}$, and denote this element as $\alpha x_{2}$. An application of the Projection Theorem yields [19]

$$
\left(z-\alpha x_{2} \mid x_{2}\right)=0, \quad \text { hence } \alpha=\frac{\left(z \mid x_{2}\right)}{\left\|x_{2}\right\|^{2}}
$$

is a maximal proper subspace, and since $x_{2}$ is orthogonal to $\Gamma_{1}$ by construction

$$
z-\alpha x_{2}=x_{\gamma}, \quad \text { for some } x_{\gamma} \in \Gamma_{1} .
$$

Furthermore, $\zeta$ is a linear functional, so

$$
\zeta(z)=\zeta\left(\alpha x_{2}+x_{\gamma}\right)=\zeta\left(\alpha x_{2}\right)=\frac{\left(z \mid x_{2}\right)}{\left\|x_{2}\right\|^{2}} \zeta\left(x_{2}\right) .
$$

The range of $\zeta$ is the real numbers, $\zeta\left(x_{2}\right)$ and $\zeta(z)$ are strictly positive, thus (6) implies that $\left(z \mid x_{2}\right)>0$. By (5) there must then exist points along the segment $(1-\lambda) x_{1}+$ $\lambda z$, contained in $C_{1}$, in the interior of $S$ and hence closer to $x_{2}$ than $x_{1}$-violating the definition of $P_{2}$. Whence, $C_{1}$ is contained in the halfspace $\{x \in H: \zeta(x) \leq 0\}$.

Now consider the hyperplane

$$
\Gamma_{2}=\Gamma_{1}+x_{2}=\left\{x \in H: \zeta(x)=\zeta\left(x_{2}\right)\right\} .
$$

Proceeding as above, and noting that $x_{1}=P_{1} x_{2}$, it may shown that $C_{2}$ is contained in the halfspace $\{x \in H: \zeta(x)$ $\left.\geq \zeta\left(x_{2}\right)\right\}$.

$\Gamma_{1}$ and $\Gamma_{2}$ are parallel by construction, thus

$$
d\left(C_{1}, C_{2}\right)=d\left(\Gamma_{1}, \Gamma_{2}\right)=\left\|x_{1}-x_{2}\right\|
$$

where $x_{1}$ was an arbitrary element of $F_{P}$, and the proof of Theorem 3 is complete.

\subsection{Asymptotically Regular Operators}

At this juncture, aspects of the structure of the composite operator $P$ and of its set of fixed points $F_{P}$ have been established, and the discussion now turns to the convergence behavior of the sequence of iterates $\left\{P_{\lambda}^{n}(P x)\right\}$, for an arbitrary $x \in H$. The requisite arguments rely to a significant extent upon the concepts of asymptotic regularity and weak convergence.

An operator $T$ in a normed linear space $X$ is said to be asymptotically regular if, for each $x \in X,\left\|T^{n+1} x-T^{n} x\right\|$ $=\left\|(I-T) T^{n} x\right\|$ converges to zero as $n \rightarrow \infty[20]$. Thus the sequence $\left\{T^{n} x\right\}$ is convergent for each $x \in X$. The following theorem asserts that any nonexpansive operator in a uniformly convex Banach Space can be "converted" to an asymptotically regular, nonexpansive operator [20], [13]. 
Theorem 4: Let $X$ be a uniformly convex Banach Space, $T$ a nonexpansive, self-mapping of $X$ with a nonempty set $F_{T}$ of fixed points. For any fixed $\lambda \in(0,1)$, define $S_{\lambda}=\lambda I$ $+(1-\lambda) T$. Then the following statements are true.

A) $F_{T}$ is the set of fixed points of $S_{\lambda}$.

B) $S_{\lambda}$ is nonexpansive.

C) $S_{\lambda}$ is asymptotically regular.

Proof of $A$ :

Suppose that $u \in X$ is a fixed point of $S_{\lambda}$. Then $S_{\lambda} u=$ $\lambda u+(1-\lambda) T u=u$, or $(1-\lambda) T u=(1-\lambda) u$. So $T u=u$, therefore, $u \in F_{T}$, and statement $\mathrm{A}$ is verified.

Proof of $B$ :

Let $x_{1}$ and $x_{2}$ be arbitrary elements of $X$, and consider $\left\|S_{\lambda} x_{1}-S_{\lambda} x_{2}\right\|$.

$$
\begin{aligned}
\left\|S_{\lambda} x_{1}-S_{\lambda} x_{2}\right\| & =\left\|\lambda\left(x_{1}-x_{2}\right)+(1-\lambda)\left(T x_{1}-T x_{2}\right)\right\| \\
& \leq \lambda\left\|x_{1}-x_{2}\right\|+(1-\lambda)\left\|T x_{1}-T x_{2}\right\| \\
& \leq \lambda\left\|x_{1}-x_{2}\right\|+(1-\lambda)\left\|x_{1}-x_{2}\right\|
\end{aligned}
$$

by the nonexpansivity of $T$. Therefore, $\left\|S_{\lambda} x_{1}-S_{\lambda} x_{2}\right\| \leq$ $\left\|x_{1}-x_{2}\right\|$ for arbitrary $x_{1}$ and $x_{2}$ in $X$ and statement $\mathrm{B}$ is established.

Proof of $C$ :

Define $x_{n}=S_{\lambda}^{n} x$ for some $x \in X$. Then

$$
\begin{aligned}
\left\|S_{\lambda}^{n+1} x-S_{\lambda}^{n} x\right\| & =\left\|S_{\lambda} x_{n}-x_{n}\right\|=\left\|(\lambda-1) x_{n}+(1-\lambda) T x_{n}\right\| \\
& =(1-\lambda)\left\|x_{n}-T x_{n}\right\| .
\end{aligned}
$$

Since $\lambda \in(0,1)$, the fact that $\left\|x_{n}-T x_{n}\right\|$ converges to zero as $n \rightarrow \infty$ will suffice to establish the asymptotic regularity of $S_{\lambda}$. Let $u$ be an arbitrary element of $F_{T}$, and note that

$$
\left\|x_{n+1}-u\right\|=\left\|S_{\lambda} x_{n}-S_{\lambda} u\right\| \leq\left\|x_{n}-u\right\|, \quad \text { all } n \geq 1
$$

by the nonexpansivity of $S_{\lambda}$. Clearly, the sequence $\left\{\left\|x_{n}-u\right\|\right\}_{n-1}^{\infty}$ is monotone nonincreasing with $n$, and it therefore converges to some nonnegative limit $d_{0}$. Hence,

$$
\left\|x_{n+1}-u\right\| \rightarrow d_{0} \quad \text { and } \quad\left\|x_{n}-u\right\| \rightarrow d_{0}, \quad \text { as } n \rightarrow \infty .
$$

Suppose that $d_{0}>0$. Suppose further that $\left\|x_{n}-T x_{n}\right\|$ does not converge to zero. Then there exists an $\epsilon>0$ such that

$$
\lim _{n \rightarrow \infty}\left\|x_{n}-T x_{n}\right\|=\lim _{n \rightarrow \infty}\left\|\left(x_{n}-u\right)-\left(T x_{n}-u\right)\right\|>\epsilon>0 \text {. }
$$

By Lemma 1, statement B, however, there exists a $\delta>0$ such that

$$
\begin{aligned}
\lim _{n \rightarrow \infty}\left\|\lambda\left(x_{n}-u\right)+(1-\lambda)\left(T x_{n}-u\right)\right\| & \\
& \leq(1-\delta)\left[\lim _{n \rightarrow \infty}\left\|x_{n}-u\right\|\right]
\end{aligned}
$$

since $\left\|T x_{n}-u\right\|=\left\|T x_{n}-T u\right\| \leq\left\|x_{n}-u\right\|$, where the $\lambda$ is that in the definition of $S_{\lambda}$. Inasmuch as $\left(x_{n+1}-u\right)=\lambda\left(x_{n}\right.$ $-u)+(1-\lambda)\left(T x_{n}-u\right)$, the convergence of $\left\|x_{n}-T x_{n}\right\|$ to some value other than zero implies that

$$
\lim _{n \rightarrow \infty}\left\|x_{n+1}-u\right\| \leq(1-\delta)\left[\lim _{n \rightarrow \infty}\left\|x_{n}-u\right\|\right]
$$

which is false, as $\left\|x_{n+1}-u\right\|$ and $\left\|x_{n}-u\right\|$ must converge to the same limit as $n \rightarrow \infty$. Therefore, by contradiction, the convergence of $\left\|x_{n}-T x_{n}\right\|$ to zero as $n \rightarrow \infty$ is established, whence $S_{\lambda}$ is asymptotically regular, completing the proof.
It is worth noting that in a slightly different context, the choice of $\lambda$ in an operator of the form $\lambda I+(1-\lambda) T$ has been shown to have a direct effect on the rate of convergence of the sequence $\left\{(\lambda I+(1-\lambda) T)^{n} x\right\}[10],[21]$, and is likely to have a similar effect here.

\subsection{Weak Convergence}

The last general notion which must be introduced prior to establishing the convergence properties of $\left\{P_{\lambda}^{n}(P x)\right\}$ is that of weak convergence. As a preface, the Reisz-Fréchet Representation Theorem [19] will be stated: "If $\zeta$ is a bounded linear functional on a Hilbert Space $H$, there exists a unique vector $y \in H$ such that for all $x \in H$, $\zeta(x)=(x \mid y)$. Furthermore, we have $\|\zeta\|=\|y\|$ and every $y$ determines a unique bounded linear functional in this way." Since the dual of a normed linear space $X$ is the space of all bounded linear functionals defined on $X$, the foregoing theorem may be interpreted as saying that a Hilbert Space is its own dual in the sense of the correspondence between bounded linear functionals defined on that space and elements of that space. As will become apparent, weak convergence involves considerations in both a normed space and its dual; and, if that space is a Hilbert Space, the Reisz-Fréchet Theorem allows the criteria for weak convergence to be phrased in terms of a single space.

Let $X^{\prime}$ denote the dual of a normed linear space $X$. A sequence $\left\{x_{n}\right\}$ in a normed linear space $X$ is said to be weakly convergent if a finite $\lim _{n \rightarrow \infty} \zeta\left(x_{n}\right)$ exists for each $\zeta \in X^{\prime} ;\left\{x_{n}\right\}$ is said to converge weakly to an element $x_{\infty} \in X$ if $\lim _{n \rightarrow \infty} \zeta\left(x_{n}\right)=\zeta\left(x_{\infty}\right)$ for each $\zeta \in X^{\prime}$ [12]. Restated in terms of the Reisz-Fréchet Theorem: A sequence $\left\{x_{n}\right\}$ in a Hilbert Space $H$ is said to be weakly convergent if a finite $\lim _{n \rightarrow \infty}\left(x_{n} \mid y\right)$ exists for all $y \in H$; $\left\{x_{n}\right\}$ is said to converge weakly to an element $x_{\infty} \in H$ if $\lim _{n \rightarrow \infty}\left(x_{n} \mid y\right)=\left(x_{\infty} \mid y\right)$ for all $y \in H$.

While strong convergence, i.e., convergence in the norm, of a sequence $\left\{x_{n}\right\}$ to an element $x_{\infty}$ in a normed space implies weak convergence of $\left\{x_{n}\right\}$ to $x_{\infty}$, the converse is not true; and examples of sequences which converge weakly to some weak limit $x_{\infty}$, but not strongly to that limit, may be constructed [19]. The following example demonstrates, however, that the notion of the weak limit is meaningful in practical situations.

This example is set in the real $L_{2}(-\infty, \infty)$ Hilbert Space. Consider a weakly convergent sequence $\left\{x_{n}\right\}_{n=1}^{\infty}$ with weak limit $x_{0}$, all in $L_{2}(-\infty, \infty)$; and suppose that the limiting behavior of $x_{n}$, as $n \rightarrow \infty$, on the compact interval $[0, T], 0<T<\infty$, is of primary interest. A countably infinite set of orthonormal basis functions, $\left\{e_{i}\right\}$, for $L_{2}[0, T]$ is then selected (for example, the basis functions for the Fourier Series, $\{(1 / T),(2 / T) \cos (2 \pi t / T)$, $(2 / T) \sin (2 / \pi t / T), \cdots, \quad(2 / T) \cos (2 n \pi t / T)$, $(2 / T) \sin (2 n \pi t / T), \cdots\})$. The functions $e_{i}(t)$ are then extended into $L_{2}(-\infty, \infty)$ by defining the new functions,

$$
e_{i}^{(2)}=\left[\begin{array}{ll}
e_{i}, & t \in[0, T] \\
0, & t \in(-\infty, 0) \cup(T, \infty) .
\end{array}\right.
$$


Now, the $L_{2}(-\infty, \infty)$ inner product of any element in $L_{2}(-\infty, \infty)$ with any one of the $e_{i}^{(2)}$ 's will equal the $L_{2}[0, T]$ inner product of that element with the corresponding $e_{i}$. Furthermore, with respect to the $L_{2}(-\infty, \infty)$ inner product, $\lim _{n \rightarrow \infty}\left(x_{n} \mid e_{i}^{(2)}\right)=\left(x_{0} \mid e_{i}^{(2)}\right)$ for all $i$. It is well known that a generalized Fourier Series of the form $\sum_{i=1}^{\infty}\left(x \mid e_{i}\right) e_{i}$, where $x \in L_{2}[0, T]$ and the $L_{2}[0, T]$ inner product is employed, converges strongly to $x$ on $[0, T]$. Since $x_{0}$, restricted to $[0, T]$, is in $L_{2}[0, T]$, strong convergence of $\left\{x_{n}\right\}$ to $x_{0}$ on $[0, T]$ has been established. In general, weak convergence in $L_{2}(-\infty, \infty)$ implies strong convergence on any compact (i.e., bounded and closed) subset of the real line.

In a somewhat analogous manner to the definition of a complete normed linear space-a Banach Space-in terms of Cauchy sequences, a normed linear space is said to be sequentially weakly complete if every weakly convergent sequence from that space converges weakly to an element of that space. It may be shown that all reflexive spaces, and thus all uniformly convex spaces, are sequentially weakly complete [12]; and this result will be employed in the proof of Theorem 5, in which the convergence properties of $\left\{P_{\lambda}^{n}(P x)\right\}$ are established.

An immediate result of the definition of a weakly convergent sequence and of a weak limit is that of the following lemma.

Lemma 5: If in a Hilbert Space $H$ the sequence $\left\{x_{n}\right\}_{n=1}^{\infty}$ is weakly convergent to $x_{0}$, then for any $x \neq x_{0}[18]$,

$$
\liminf _{n \rightarrow \infty}\left\|x_{n}-x\right\|>\liminf _{n \rightarrow \infty}\left\|x_{n}-x_{0}\right\| \text {. }
$$

Proof: By definition of the norm, both limits are finite. Consider,

$$
\begin{aligned}
\left\|x_{n}-x\right\|^{2} & =\left\|x_{n}-x_{0}+x_{0}-x\right\|^{2} \\
& =\left\|x_{n}-x_{0}\right\|^{2}+2 \operatorname{Re}\left(x_{n}-x_{0} \mid x_{0}-x\right)+\left\|x_{0}-x\right\|^{2}
\end{aligned}
$$

Since $x_{0}$ is the weak limit of $\left\{x_{n}\right\}_{n=1}^{\infty},\left(x_{n} \mid x_{0}-x\right) \rightarrow$ $\left(x_{0} \mid x_{0}-x\right)$ as $n \rightarrow \infty$, and $\left(x_{n}-x_{0} \mid x_{0}-x\right) \rightarrow 0$ as $n \rightarrow \infty$. $\left\|x_{0}-x\right\|>0$ and the Lemma is established.

One more lemma will be required for the proof of Theorem 5; and, towards this end, the notion of a demiclosed operator will now be introduced. Let $C$ be a closed convex set in a Banach Space $X$, and $A$ a mapping from $C$ into $X$. If for any sequence $\left\{x_{n}\right\}$ in $C$ with weak limit $x_{0}$ in $C$, the strong convergence (i.e., in norm) of the sequence $\left\{A x_{n}\right\}$ to some $y_{0} \in X$ implies that $A x_{0}=y_{0}$, then the operator $A$ is said to be demiclosed [18].

Lemma 6: In a Hilbert Space $H$, for every nonexpansive mapping $T$ of $C$, a closed convex set, into $H$, the mapping $(I-T)$ is demiclosed [18].

Proof: Let $\left\{x_{n}\right\}$ be a weakly convergent sequence in $C$ with weak limit $x_{0} \in C$, and let the sequence $\left\{x_{n}-T x_{n}\right\}$ converge to some $y_{0} \in H$. By the nonexpansivity of $T$,

$$
\liminf _{n \rightarrow \infty}\left\|x_{n}-x_{0}\right\| \geq \liminf _{n \rightarrow \infty}\left\|T x_{n}-T x_{0}\right\| .
$$

Noting that $T x_{n}=x_{n}-y_{0}$ in the limit as $n \rightarrow \infty$, the preceding inequality may be rewritten as

$$
\liminf _{n \rightarrow \infty}\left\|x_{n}-x_{0}\right\| \geq \liminf _{n \rightarrow \infty}\left\|x_{n}-y_{0}-T x_{0}\right\|
$$

and since $\left\{x_{n}\right\}_{n=1}^{\infty}$ converges weakly to $x_{0}, x_{0}=y_{0}+T x_{0}$ or $(I-T) x_{0}=y_{0}$, by virtue of Lemma 5 . Therefore, $(I-T)$ is demiclosed.

\subsection{Convergence Theorem}

Our final theorem may now be stated and proven.

Theorem 5: Let $C$ be a bounded closed convex set in a Hilbert Space $H$, and let $T$ be a nonexpansive, asymptotically regular mapping of $C$ into itself with a nonempty set of fixed points $F_{T}$. Then, for any $x \in C$, the sequence of iterates $\left\{T^{n} x\right\}$ is weakly convergent to an element of $F_{T}$ [18].

Proof: The sequence $\left\{T^{n} x\right\}$ will first be shown to be weakly convergent. Let $u$ be an element of $F_{T}$, by the nonexpansivity of $T$ it is true that

$\left\|T^{n} x\right\|-\|u\| \leq\left\|T^{n} x-u\right\| \leq\left\|T^{n-1} x-u\right\| \leq \cdots \leq\|x-u\|$.

Thus $\left\|T^{n} x\right\| \leq\|x-u\|+\|u\|$ for all $n$. Let $\zeta$ be any bounded linear functional defined on $H$, and let $y \in H$ be the corresponding element of $H$ in the sense of the Reisz-Fréchet Theorem. Then $\|y\|=\|\zeta\|<\infty$, and

$$
\begin{aligned}
\lim _{n \rightarrow \infty}\left|\zeta\left(T^{n} x\right)\right| & =\lim _{n \rightarrow \infty}\left|\left(T^{n} x \mid y\right)\right| \\
& \leq \lim _{n \rightarrow \infty}\left\|T^{n} x\right\| \cdot\|y\| \\
& \leq(\|x-u\|+\|u\|) \cdot\|y\| \\
& <\infty
\end{aligned}
$$

where the Cauchy-Schwarz Theorem has been invoked. Hence $\left\{T^{n} x\right\}$ is weakly convergent and has its weak limit in $H$, since Hilbert Spaces are weakly sequentially complete.

Recalling the definitions of $d(u)$ and $F_{\Delta}$ from Corollary 1 , let $F_{\Delta}=\left\{u_{0}\right\}$ which implies that $d\left(u_{0}\right)=\Delta$, where the $x$ of $d(\cdot)$ is that of the statement of this Theorem. Through a proof by contradiction, $\left\{T^{n} x\right\}$ will be shown to converge weakly to $u_{0}$.

Suppose that $\left\{T^{n} x\right\}$ converges weakly to some $z \in H$, $z \neq u_{0} . T$ is asymptotically regular, by definition, and the sequence $\left\{T^{n} x-T^{n+1} x\right\}=\left\{(I-T) T^{n} x\right\}$ must therefore converge strongly to the origin as $n \rightarrow \infty$. By Lemma 6 , $(I-T)$ is demiclosed, so it must be the case that $(I-T) z$ $=\phi$ as well. Hence, $z \in F_{T}$. The result of Lemma 5 is then

$$
\Delta=d\left(u_{0}\right)=\lim _{n \rightarrow \infty}\left\|T^{n} x-u_{0}\right\|>\lim _{n \rightarrow \infty}\left\|T^{n} x-z\right\|=d(z)
$$

since the limit infimum of a sequence equals the limt of the sequence if that limit exists (the existence of the above limits was established in the discussion preceding Corollary 1). This inequality violates the definition of $\Delta$, generating the necessary contradiction and completing the proof.

\subsection{Synoposis}

Each of the results of the preceding seven sections was presented in the most general context possible, with the intent of providing points of departure for further study 
and development of the iterative synthesis technique of this paper and of other similar techniques. Those results will now be reiterated explicitly in terms of signal synthesis in the presence of inconsistent constraints.

The setting is a Hilbert Space $H$ defined over the scalar field of real numbers. $C_{1}$ and $C_{2}$ are disjoint, closed convex sets in $H$-each one composed of the class of signals satisfying some given constraint, or set of constraints. Furthermore, $C_{1}$ is bounded. Lemma 2 guarantees that the respective nearest point maps $P_{1}$ and $P_{2}$ exist, and that they yield unique points in these sets for every $x \in H$.

The composite operator $P=P_{1} \circ P_{2}$ is then nonexpansive by Theorem 1; and Theorem 2 assures that the fixedpoint set $F_{P}$ of $P$, and hence the set of dual fixed points $D_{P}$, are not empty. Theorem 3 warrants that $\left\|x_{1}-x_{2}\right\|=$ $d\left(C_{1}, C_{2}\right)$ for all $\left(x_{1}, x_{2}\right) \in D_{P}$.

Theorem 4 states that any operator of the form $P_{\lambda}=\lambda I$ $+(1-\lambda) P, \lambda \in(0,1)$ is a nonexpansive asymptotically regular operator with $F_{P}$ as its set of fixed points. Theorem 5 is thus applicable, and the sequence of iterates $\left\{P_{\lambda}^{n} x\right\}$ will converge weakly to an element of $F_{P}$ for any $x \in C_{1}$. More generally, $\left\{P_{\lambda}^{n}(P x)\right\}$ will converge weakly to an element of $F_{P}$ for any $x \in H$, as $P x \in C_{1}$.

It is worth noting that $P_{1}, P_{2}$ and $P$ are not, at any point in this section, assumed to be linear-this synthesis technique will always be appropriate, so long as $C_{1}$ and $C_{2}$ are of correct form.

\section{EXAMPLE}

As an example of signal synthesis in the presence of an inconsistent set of constraints, the technique developed in Section II will now be applied to the generation of a data window for spectral estimation. A data window is a time function which is necessarily of finite duration, and whose spectral energy is, ideally, concentrated about the origin in the frequency domain. By virtue of its finite extent in time, the window will be of infinite bandwidth; and, in practice, the design of such a window may be viewed as an attempt to attain a narrow, high amplitude, spectral concentration about the frequency origin, while simultaneously minimizing that window's spectral energy for nonzero frequencies - in the parlance of spectral estimation, the desire is to maximize resolvability while minimizing leakage.

The two disjoint closed convex sets from $L_{2}(-\infty, \infty)$, to which the synthesis technique of this paper will be applied, have been selected as follows. $C_{2}$ will be the set of all time signals which identically zero outside of the interval [ $-T / 2, T / 2]$, where $T$ is the duration of the desired data window. $C_{1}$ will be the set of all signals whose Fourier transforms are real, nonnegative, bandlimited to some frequency $\Omega$, and have a prescribed area $\rho$. Elements of $C_{1}$ will be real, even time functions, and their projections into $C_{2}$ will thus be even as well. Denoting the Fourier transform of some $f(t) \in L_{2}(-\infty, \infty)$ as $F_{1}(\omega)+j F_{2}(\omega)$, where $F_{1}$ and $F_{2}$ are real-valued functions of $\omega$, Youla has shown that the projection of $f(t)$ into $C_{1}$ is given by $\mathscr{F}^{-1}$ $\left\{r_{\Omega}(\omega)\left(F_{1}(\omega)+c\right)^{+}\right\}[10]$, where $\mathscr{F}^{-1}$ denotes the inverse
Fourier transform,

$$
\begin{aligned}
r_{\Omega}(\omega) & =\left[\begin{array}{ll}
1, & \omega \in[-\Omega, \Omega] \\
0, & \omega \notin[-\Omega, \Omega]
\end{array}\right. \\
(G(\omega))^{+} & =\left[\begin{array}{ll}
G(\omega), & G(\omega) \geq 0 \\
0, & G(\omega)<0
\end{array}\right.
\end{aligned}
$$

and where the real constant $c$ is chosen such that

$$
\int_{-\Omega}^{\Omega}\left(F_{1}(\omega)+c\right)^{+} d \omega=\rho .
$$

The projection of some $f(t) \in L_{1}(-\infty, \infty)$ into $C_{2}$ is clearly $r_{T / 2}(t) f(t)$, where

$$
r_{T / 2}(t)=\left[\begin{array}{ll}
1, & t \in\left[-\frac{T}{2}, \frac{T}{2}\right] \\
0, & t \notin\left[-\frac{T}{2}, \frac{T}{2}\right]
\end{array} .\right.
$$

It should be noted, before proceeding with this example, that there are many available techniques for estimating the spectral content of a signal. The generation of such an estimate by the transformation of the product of an observed segment of the signal and a data window is but one method. The intent of this discussion is not an in-depth analysis of spectral estimation-the reader is referred to a paper by Kay and Marple [22] for an overview of the subject, and to a paper by Harris [23] on the topic of data windows.

An iterative technique, such as the one of this paper, must be implemented on a digital computer to be of practical worth. Time and frequency axes, as well as function amplitudes, must be discretized. Since the sets $C_{1}$ and $C_{2}$ were defined in terms of $L_{2}(-\infty, \infty)$, instead of $l_{2}(-\infty, \infty)$, all the usual caveats concerning approximations to the continuous world by the discrete one must be heeded. An attempt has been made to minimize aliasing effects by setting the Nyquist Frequency to be more than seventy times the value of $\Omega$ in the definition of $C_{1}$, and any integrals which must be approximated will be calculated using the trapezoidal rule. It is assumed that a discrete approximation to the projection operator corrcsponding to $C_{1}$ will be appropriate, although, for reasons of practicality, the area $\rho$ will now become a range of areas $\rho \pm \Delta$, where $\Delta \leq 10^{-5} \rho$. Perhaps the most important aspect of this discrete approximation is that the use of a finitelength Discrete Fourier Transform (DFT) will implicitly generate periodic functions.

The specific example to be presented is that of the design of a 64 point data window for spectral estimation. It is assumed that all signals of interest have negligible frequency content above $10 \mathrm{~Hz}$-the selected Nyquist Frequency, $F_{N}$. The sampling frequency, $F_{S}$, will be $20 \mathrm{~Hz}$, yielding a sampling period $T_{S}$ of $0.05 \mathrm{~s}$ and a spacing of $\Delta_{f}^{(1)}=F_{S} / 64$ $=0.3125 \mathrm{~Hz}$ for the frequency samples of the associated DFT.

The data window will be designed on a 1024-point sequence with the same spacing in time, $T_{S}$, as the 64 point 
sequence, and, hence, with a spacing of $\Delta_{f}^{(2)}=F_{S} / 1024=$ $0.01953125 \mathrm{~Hz}$ for the frequency samples of the associated DFT. Now, $\Delta_{f}^{(2)} / \Delta_{f}^{(1)}=16$; and the advantage of this factor of 16 density increase in frequency samples for the design sequence, over the sequence whose length is that of the data window, will be that $C_{1}$ may be meaningfully defined with values of $\Omega$ less than $\Delta_{f}^{(1)}$.

In order to provide the most realistic emulation of the continuous case, the time origin for the 1024-point sequence was defined to be the 513th point of that sequence. Any sequence of 1024 points which is real and displays even symmetry about the 513th point will have a real and even-about the frequency origin-DFT. With the time origin so defined, the span of the truncation window $r_{T / 2}(t)$ will be an odd number of points. In what follows, then, the $C_{2}$ functions which have been generated by the iterative synthesis technique will have been produced by a 65-point rectangular window of height one, centered at point 513. The data window, whose performance will be subsequently evaluated, will be composed of the final $C_{2}$ sequence from the point whose time abscissa is $-T / 2$ up to, but excluding, the point whose time abscissa is $T / 2$ yielding a sequence of length 64 .

A modular, Fortran 77, program named CONVEX was developed to carry out iterations of the form $\left\{P_{\lambda}^{n}\right\}$. The program was supplied with the projection routines for the $C_{1}$ and $C_{2}$ of this section. $C_{1}$ was parameterized as follows: $\rho=1.3 \pm 1.3 \times 10^{-5}$, and $\Omega=7 \Delta_{f}^{(2)}=7 / 16 \Delta_{f}^{(1)}=0.13671875$ $\mathrm{Hz}$. The single parameter for $C_{2}, T / 2$, was set to $32 T_{S}=1.6$ s. The fixed points of $P$ were specified to lie in $C_{1}$, thus $P=P_{1} \circ P_{2} ;$ and the value of $\lambda$, in the definition of $P_{\lambda}$, was chosen to be $10^{-3}$. For this example, CONVEX was instructed to carry out 50 iterations and to make the results of the 3rd and 50th iterations available for inspection.

To aid in the evaluation of the convergence properties of the sequence of iterates, the following measure of convergence was defined

$$
\epsilon(n)=\frac{\left\|P_{\lambda}^{n}(P x)-P_{\lambda}^{n-1}(P x)\right\|}{\left\|P_{\lambda}(P x)-P x\right\|}, \quad n=1,2, \cdots .
$$

Since $P_{\lambda}$ is nonexpansive, $\epsilon$ will be a monotone nonincreasing function of $n$. Moreover, the asymptotic regularity of $P_{\lambda}$ would lead one to expect $\epsilon$ to be a decreasing function for sufficiently large $n$.

The starting point for the iterations, the " $x$ " of $\left\{P_{\lambda}^{n}(P x)\right\}$, is displayed in Fig. 3. This function is neither an element of $C_{1}$ nor of $C_{2}$. In the process of developing CONVEX, it was observed that the point to which the sequence of iterates converged was essentially invariant with respect to its starting point.

Figs. 4 and 5 are plots of $\epsilon(n)$ versus $n$ on two different ordinate scales. The technique is obscrved to converge rapidly. With these two sets, convergence is typically achieved within forty iterations for a wide range of time-bandwidth products generated by particular parameterizations of $C_{1}$ and $C_{2}$. The oscillatory behavior of $\epsilon$,
INITIAL FUNCTION IN TIEE (SEC) DOMAIN A TER NON ITERATIONS

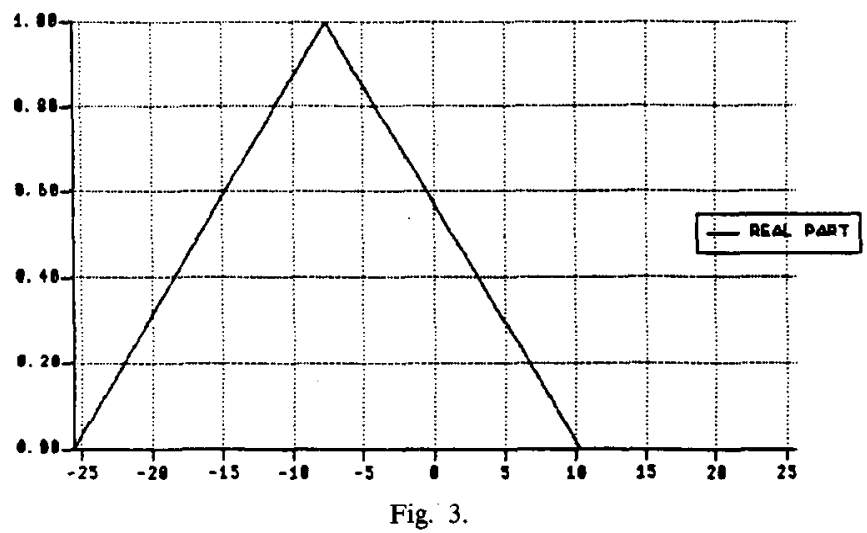

NORMALIZED EPSILON VS. ITERATIONE FOR A TOTA OF MOSE ITEMATIONS

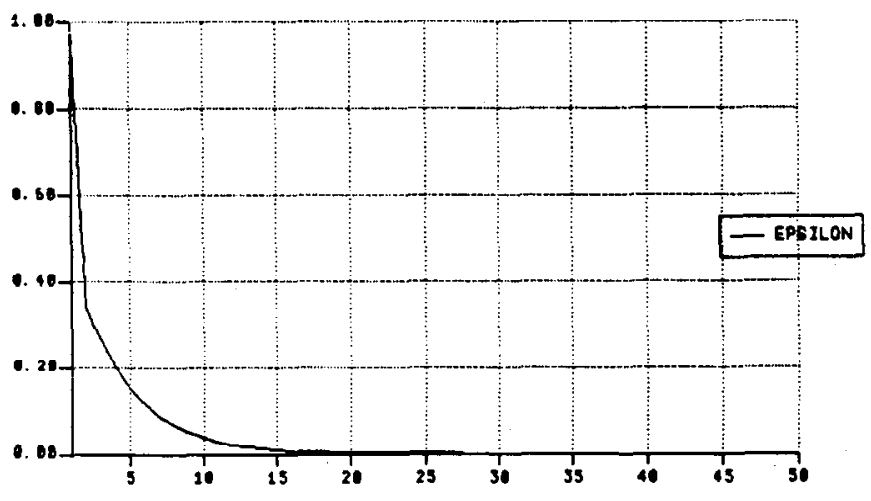

Fig. 4.

MORML IZED EPSILON YS. ITERATIONG FOR A TOTK OF OASE ITERATIONS

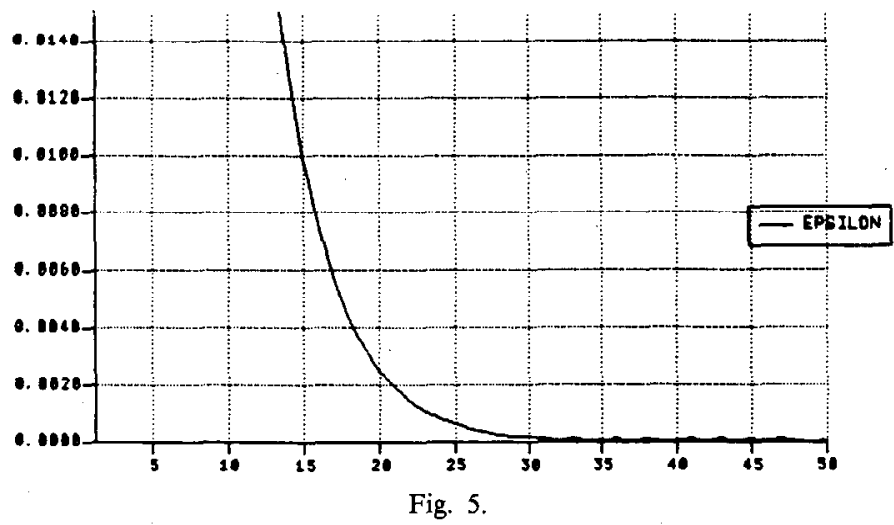

above $n=32$, may be ascribed to the fact that the $\rho$ for $C_{1}$ was defined to be a range of values, rather than a single value.

Figs. 6 and 7 depict the $C_{1}$ function after three iterations in the time and frequency domains, respectively. In the time domain, the function is observed to be radically different from the initial function of Fig. 3, and to be periodic - a result of time discretization and the use of the DFT in the associated projection routine. The time function also displays even symmetry about the time origin, as 
BET I FUNCTJON IN TIRE (BEC) DOMNIN N TER MEN ITERATIONS

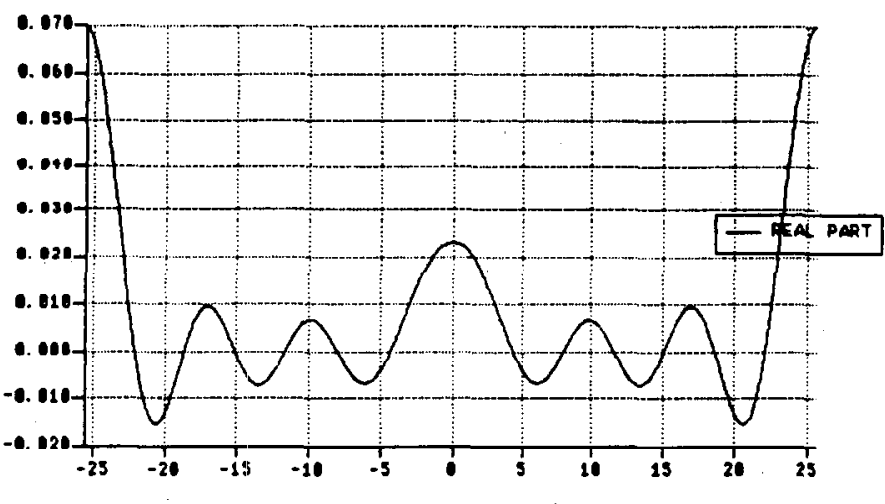

Fig. 6.

SET 1 FUNCTION IN FREQUENCY IMZ DOMAIN AFTER MNS ITERATIONS

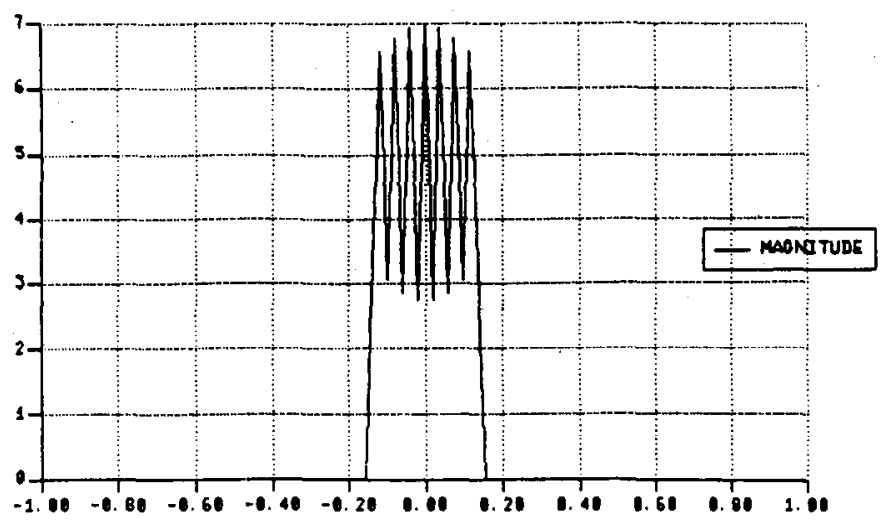

Fig. 7.

SET 2 FUNCTION IN TIME (SEC) DOMAIN AFTER 000S ITERATIONS

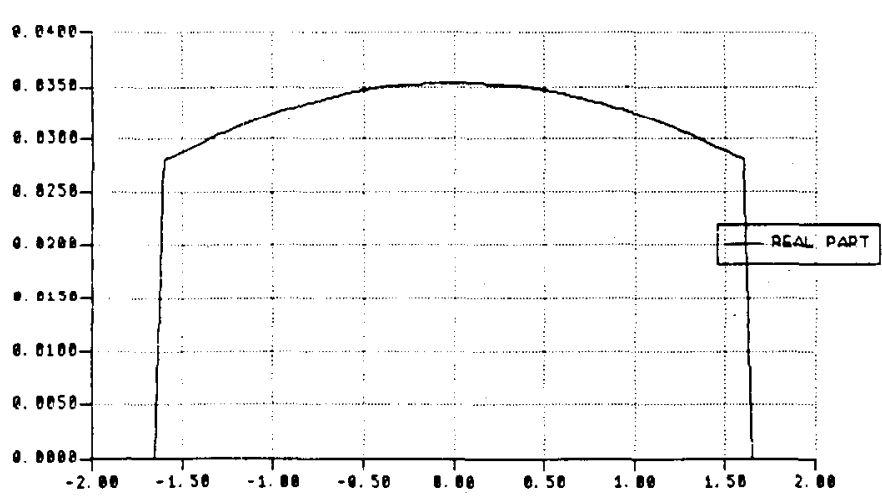

Fig. 8.

is expected. Fig. 7 shows that the function is composed of 6 cosinusoids and a constant. Although the inclusion of a component at $7 \Delta_{f}^{(2)} \mathrm{Hz}$ is allowed, its presence is difficult to observe in either the time or frequency plots, as the time plot displays a total of twelve peaks and dips. One should note that the definition of $C_{1}$ implies that Fig. 7 is the entire DFT of Fig. 6, since elements of $C_{1}$ have real, positive transforms.

The $C_{2}$ function, after three iterations, is displayed in Fig. 8, while its DFT appears in Fig. 9. The time function is the product of $r_{T / 2}(t)$ and the $C_{1}$ function after two iterations. The function is evenly symmetric about the
SET 2 FUNCTION IN FREQUENCY IHZI DOMAIN AFTER MON ITERATIONS

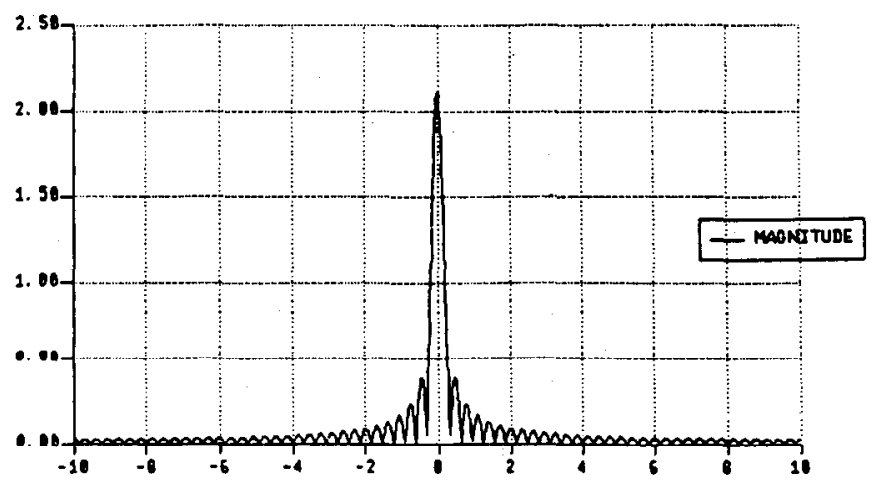

Fig. 9.

SET 1 FUNCTION IN TINE (SEC) DOMAM F TER DOSE ITERATIONS

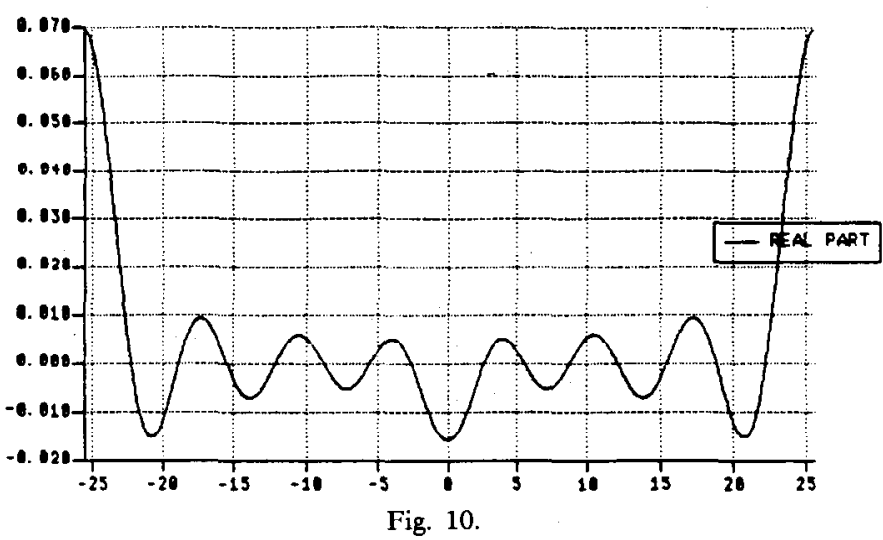

SET I FUNCTION IN FREOUENCY IHZ DOMAIN AFTFR MISE ITERATIONS

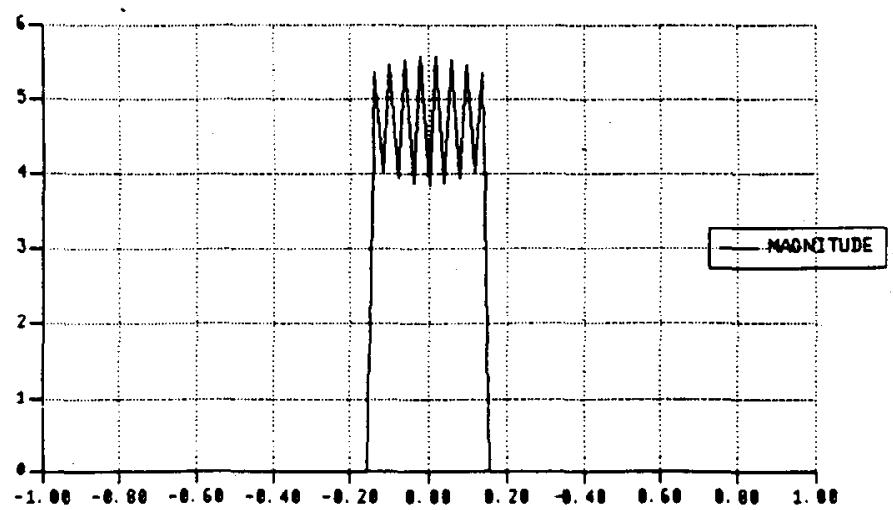

Fig. 11.

origin and real. Its DFT is, therefore, real. The appearance of Fig. 9 is typical of the result of the application of a time truncation operator to a bandlimited signal.

Figs. 10 and 11 represent the $C_{1}$ function, after fifty iterations, in the time and frequency domains, respectively. The most noticeable difference between this time function and the $C_{1}$ function after three iterations is the inversion of the central lobe about the time points $-4 \mathrm{~s}$ and $4 \mathrm{~s}$. The function now has a total of 14 peaks and dips, and, as is to be expected, the frequency corresponding to $7 \Delta_{f}^{(2)} \mathrm{Hz}$ is now in evidence in the frequency plot. The ordering of the relative amplitudes of the various frequency components 
SET I FUNCTION IN TIFE (SEC) DOMAIN AFTER OSS ITERATIONS

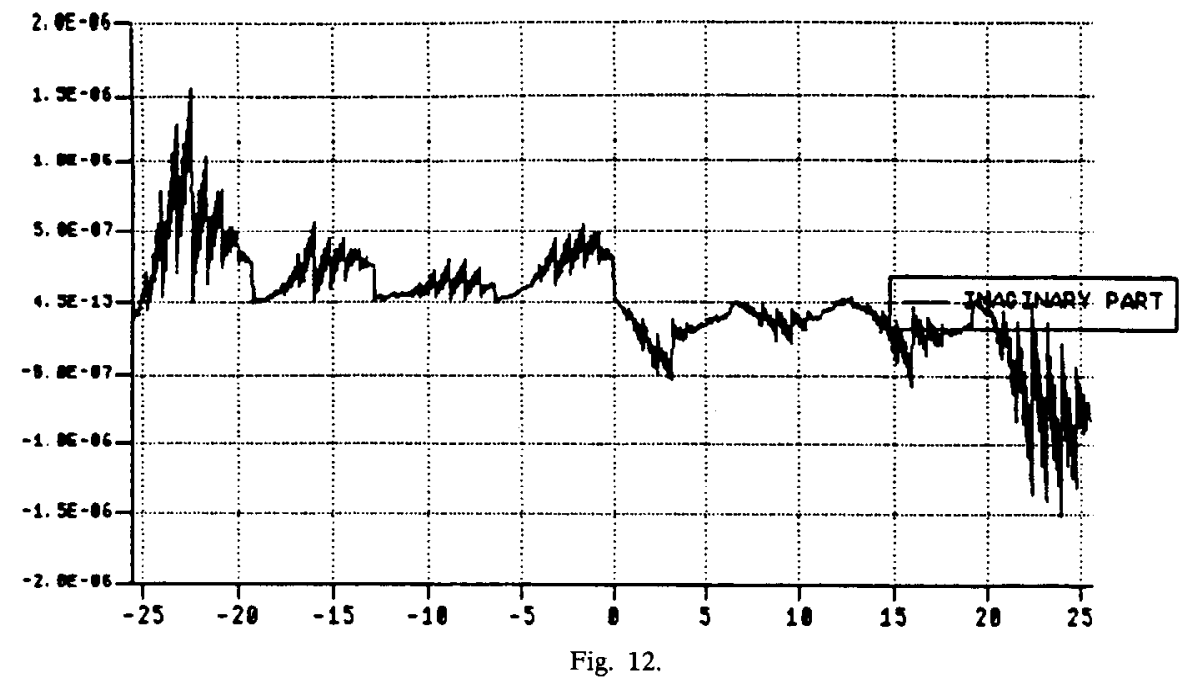

SET 2 FUNCTION IN TIFE (SEC) DOMAIN, AFTER NOSA ITERATIONS

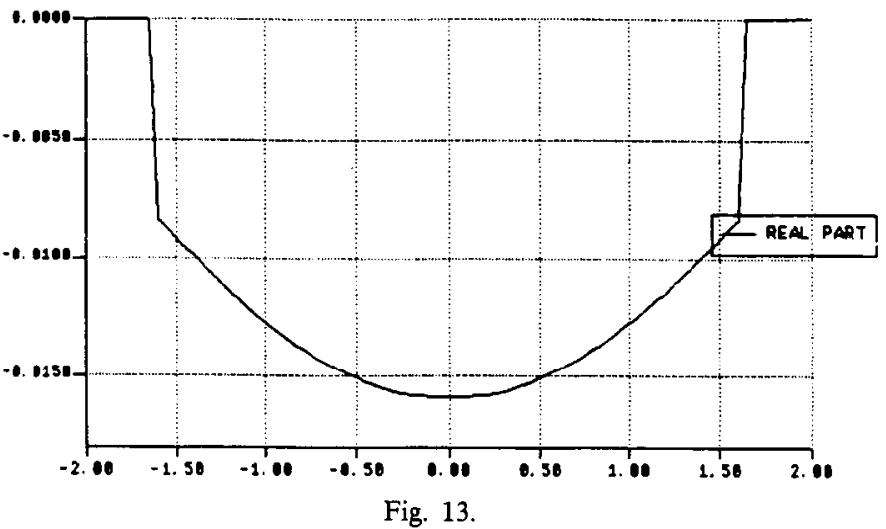

has also been reversed from that at the third iteration; and the relative amplitudes of these components has equalized somewhat.

As evidence of the propriety of the discrete implementation of the iterative technique in the program CONVEX, Fig. 12 -the imaginary part of the $C_{1}$ function after 50 iterations-has been included in this section. The peak amplitude in Fig. 12 is more than $90 \mathrm{~dB}$ below the peak of the corresponding real function, Fig. 10, and is well within the limits of computational "noise". The fact that this data appears to have some structure is not surprising in light of the iterative nature of the synthesis technique.

Figs. 13 and 14 depict the $C_{2}$ function, after 50 iterations, in the time and frequency domains, respectively. The primary differences between this time function and the $C_{2}$ function after three iterations are its negativity-corresponding to the inversion of the central lobe in the fiftieth iterate in $C_{1}$-and its increased curvature. In the frequency domain, the key change is the increased ratio of the main lobe amplitude to the amplitudes of the sidelobes. For the third $C_{2}$ iterate, the ratio of the main lobe's amplitude to that of the first sidelobe is approximately 6.0 , while for the 50th iteration this ratio is about 7.2.
SET 2 FUNCTION IN FREQUENCY (HZ) DOMAIN A TER NOSO ITERATIONS

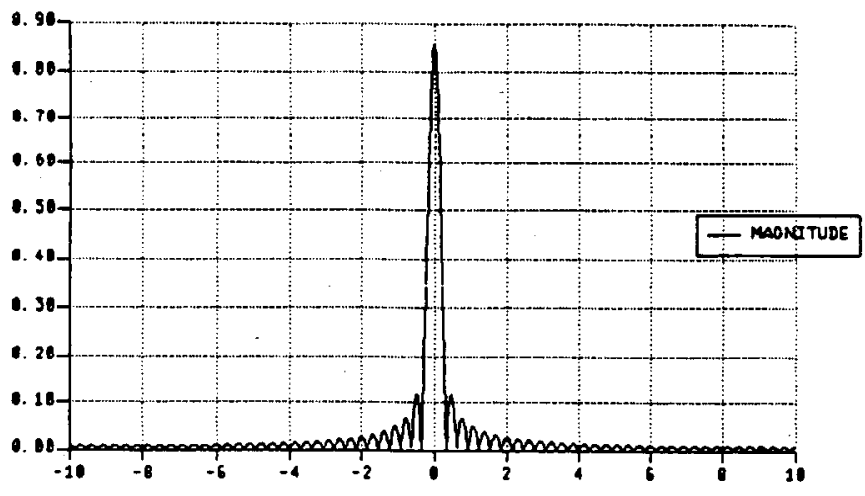

Fig. 14.

Fig. 15 is the magnitude of the DFT of the 50th $C_{2}$ iteration, on an expanded abscissa; and Fig. 16 is the magnitude of the DFT of a 65-point rectangular window, whose amplitude in time was normalized to yield the same main frequency lobe amplitude. The main frequency lobe of the $C_{2}$ function appears to be slightly wider than that of the rectangular window. The side lobe amplitudes of the $C_{2}$ function are, however, approximately $2 / 3$ of the values of those of the rectangular window.

The efficacy of the fiftieth $C_{2}$ iterate as a data window will now be evaluated. As stated earlier, the window will be formed by retaining the values of this $C_{2}$ function whose time abscissas run from $-T / 2$ up to, but excluding, the point corresponding to time $T / 2$. The performance of this window will be compared with that of the rectangular window, the Hanning window and the Hamming window [24]. These particular windows were selected because they form a representative collection of frequently used windows. The rectangular window is known for its good resolvability - narrow main lobe-and poor leakage properties-high side lobe amplitudes. The Hanning window has just the opposite properties-low leakage, but poor resolvability. The Hamming window attempts to attain a balance of 
SET 2 FUNCTION IN FREQUENCY IHZ DOMAIN NTER ONSO ITERATIONS

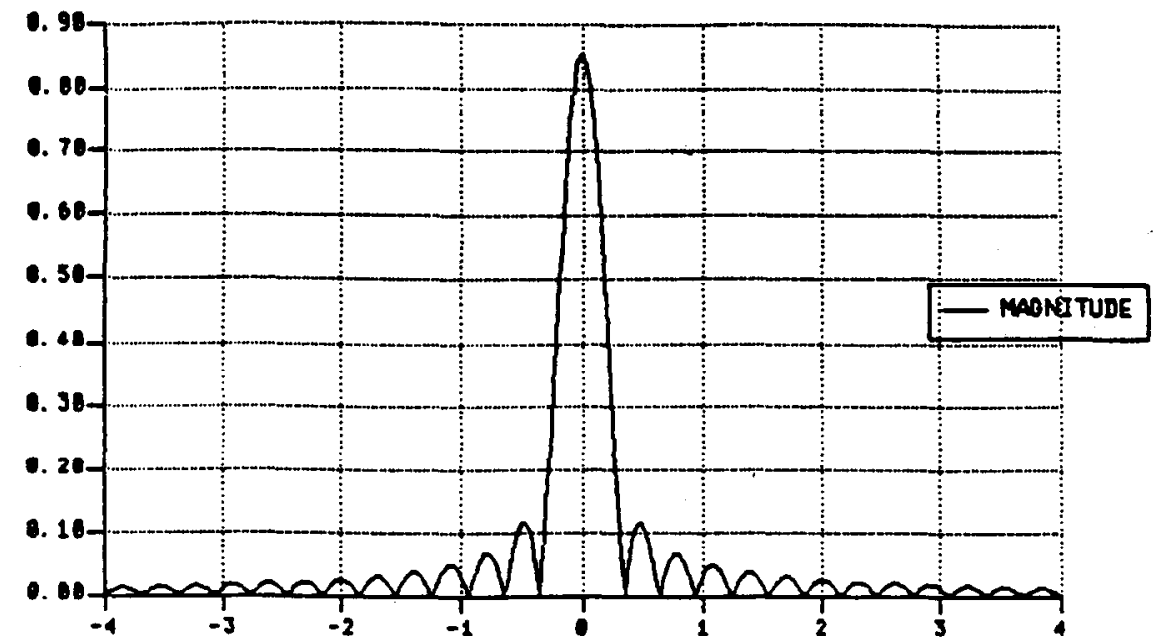

Fig. 15.

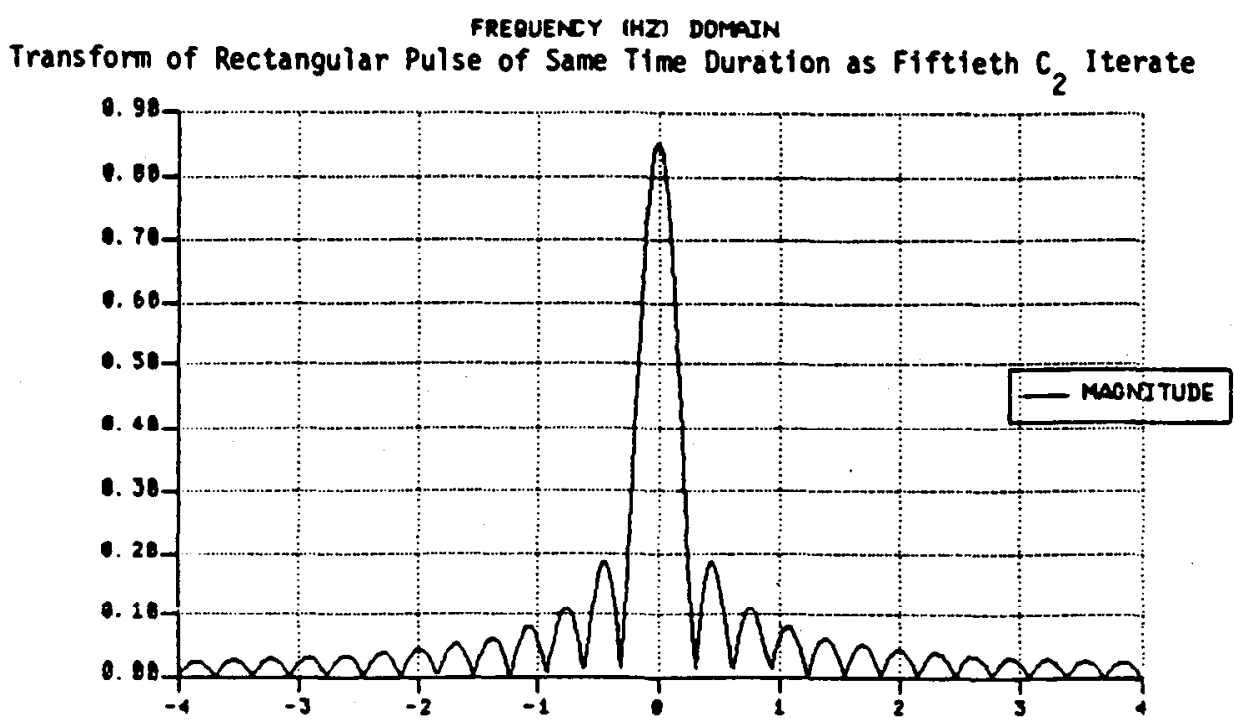

Fig. 16.

leakage and resolvability and is, in fact, a weighted average of the Hanning and rectangular windows. For a sequence of length $N$, the Hanning and Hamming windows are, respectively, defined to be

$$
\frac{1}{2}\left[1-\cos \left(\frac{2 \pi n}{N-1}\right)\right], \quad 0 \leq n \leq N-1
$$

and

$$
0.54-0.46 \cos \left(\frac{2 \pi n}{N-1}\right), \quad 0 \leq n \leq N-1 .
$$

To aid in comparisons, the windows were normalized so that their main spectral lobes were of equal amplitude.

The four windows will first be compared on the basis of leakage performance. By applying the windows to a single sinusoid of frequency $16.5 \Delta_{f}^{(1)}=5.15625 \mathrm{~Hz}$, with unit amplitude, the main spectral lobe for each window will be centered between adjacent frequency sample points of the 64-point DFT, and the peaks of the side lobes will be approximately aligned with the sample points. Figs. 17-20 depict this example for the generated window, the rectan-
TRAGGORM IN FREDUENCY (HZ) DOMAIN OF DATA WJNOOLED IY MUEATIVE RESUT
Leakage Perfomance

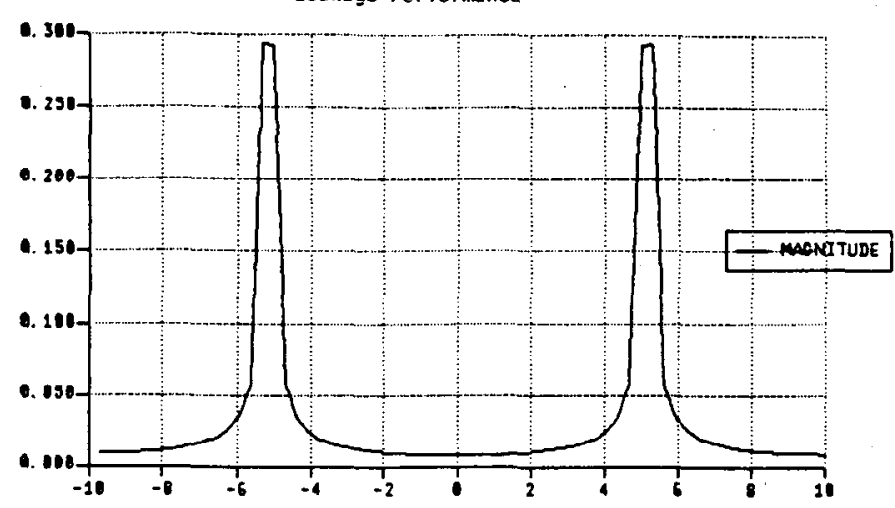

Fig. 17.

gular window, the Hanning and Hamming windows, respectively. A comparison of the peak amplitudes among the four plots reveals that the Hanning window most effectively confines the energy of the sinusoid, followed by the Hamming, the generated and the rectangular windows, 
RECTAOULA WINDOLED TRAGFORM OF DATA IN FREOUENCY OQI DOMADN Leakage Performance

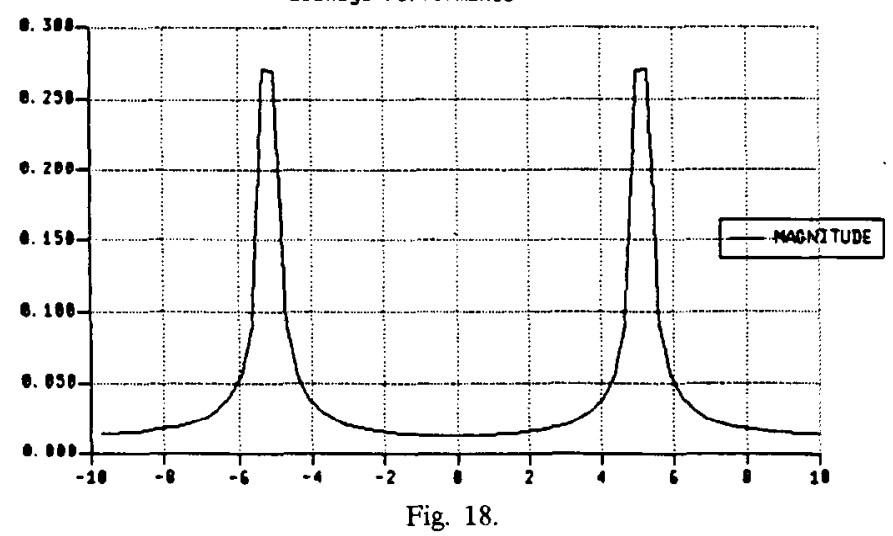

MANING WINDOUED TRMSFDRM OF DATA IN FREQUERCY (MZ) DOMADN Leakage Performance

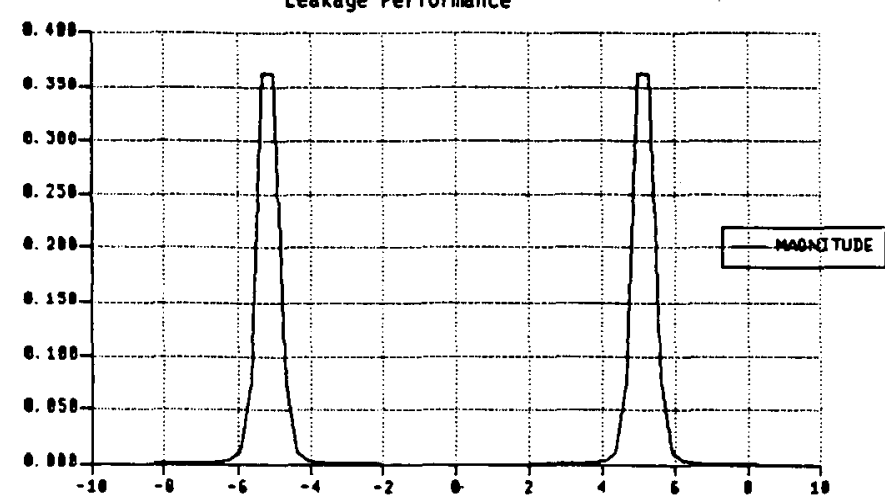

Fig. 19.

HAMIND WINDOLED TRANSFORM OF DATA IN FREQUENCY (MZ) DOMAN Leakage Performance

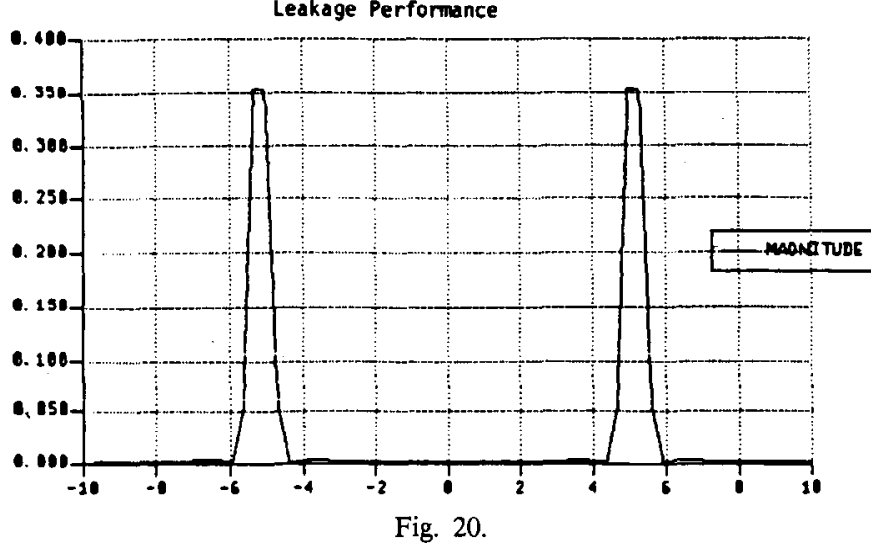

respectively. On the basis of the lobe widths at the 0.050 amplitude level, the generated window may be observed to have better performance, with respect to leakage in the vicinity of the main lobe, than the rectangular window. In this respect, the generated window may also be seen to have performance equal to that of the Hanning window, and nearly so good as that of the Hamming window.

The resolvability of the various windows is contrasted in Figs. 21-24 (generated, rectangular, Hanning and Hamming, respectively). The windows were applied to two closely spaced, unit amplitude, sinusoids-one at $10.62 \Delta_{f}^{(1)}$ $=3.31875 \mathrm{~Hz}$ and the other at $11.38 \Delta_{f}^{(1)}=3.55625 \mathrm{~Hz}$. The generated window may be observed to perform nearly so

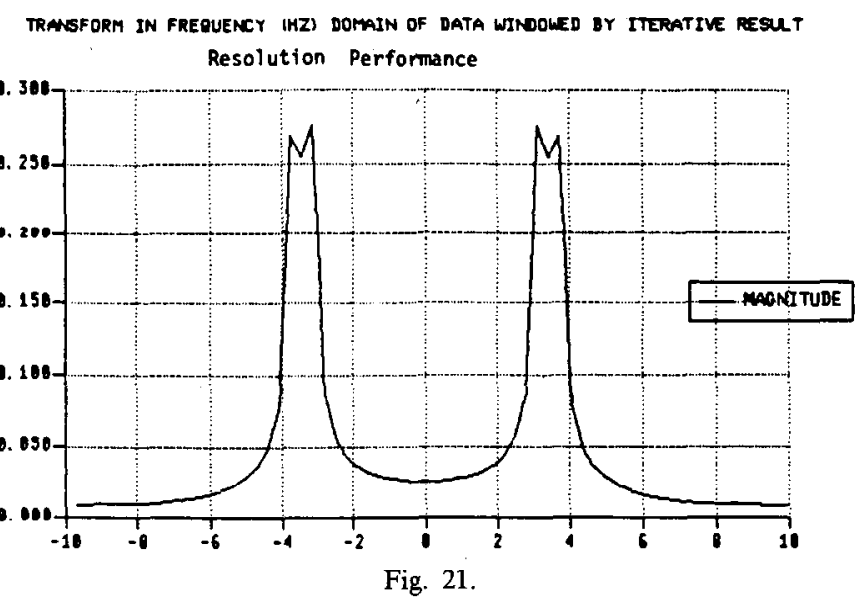

RECTMDULAR WINDOLED TRANGFORM OF DATA IN FREDUENCY OEI DOMADN Resolution Performance

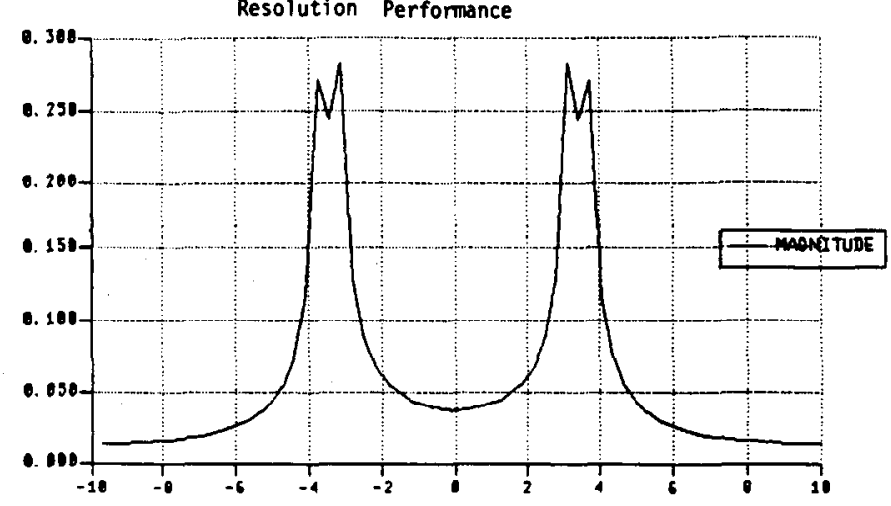

Fig. 22.

MANINO WINDOWED TRMSFORM OF DATA IN FREQUENCY (HZ) DOMAN Resolution Performance

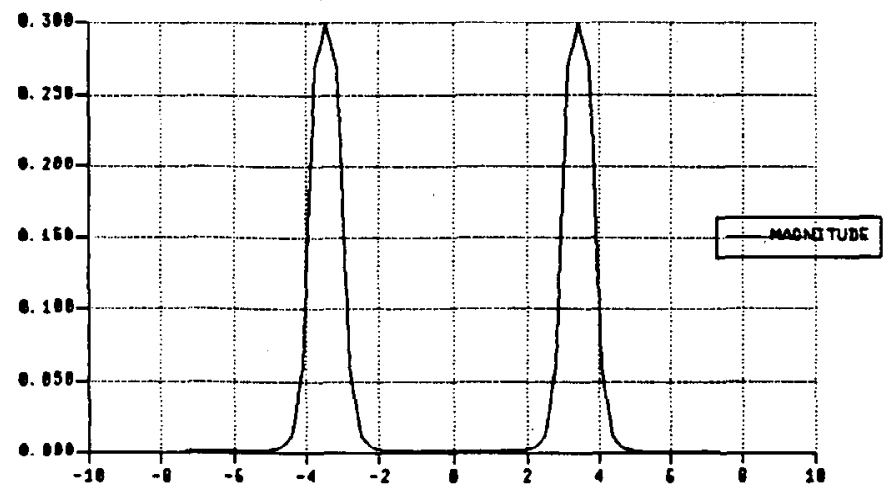

Fig. 23.

MMTIINO WINDOWED TRMGFORM OF DATA IN FREQUENCY IHD DOMADN Resolution Performance

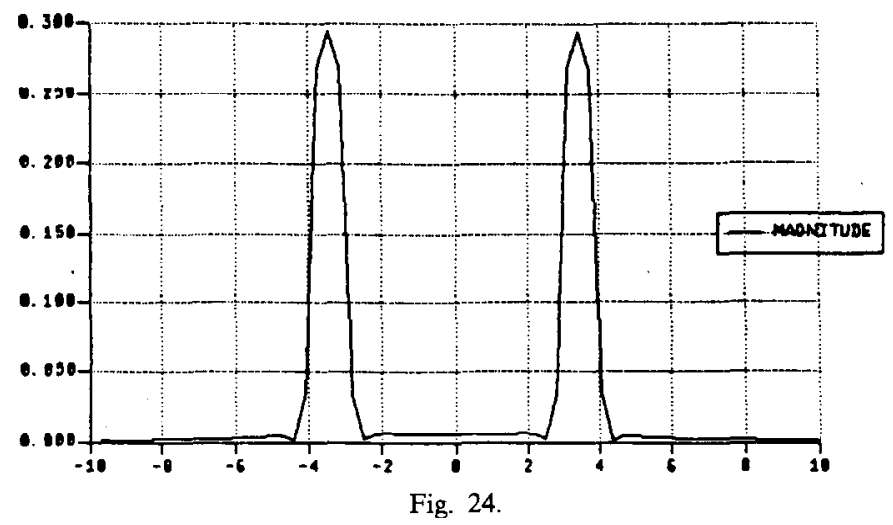


TRASFORM IN FREQUENCY IHZ) DOMAIN OF DATA WINOOWED OY ITERATIVE RESUT Combined Resolution and Leakage Performance

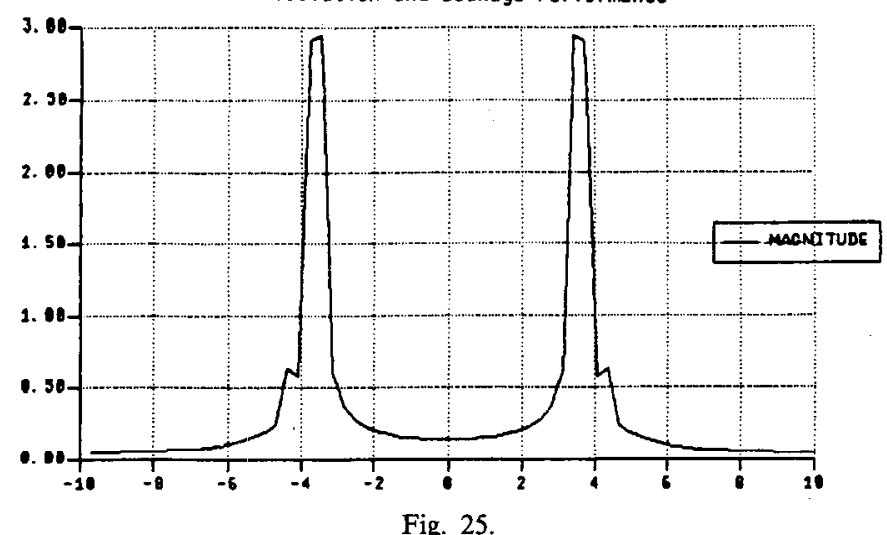

RECTNAULAR WINDOUED TRAAGFORM OF DATA IN FREQUENCY OEI DOMADN Combined Resolution and Leakage Performance

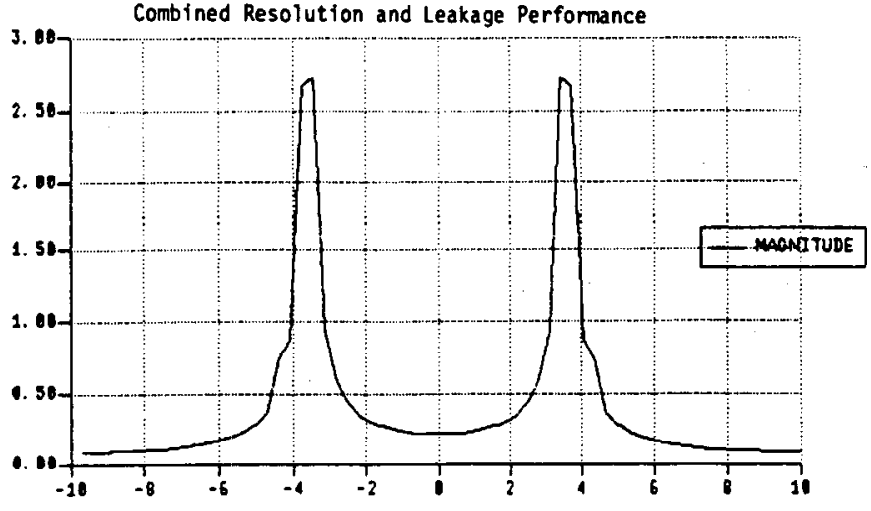

Fig. 26.

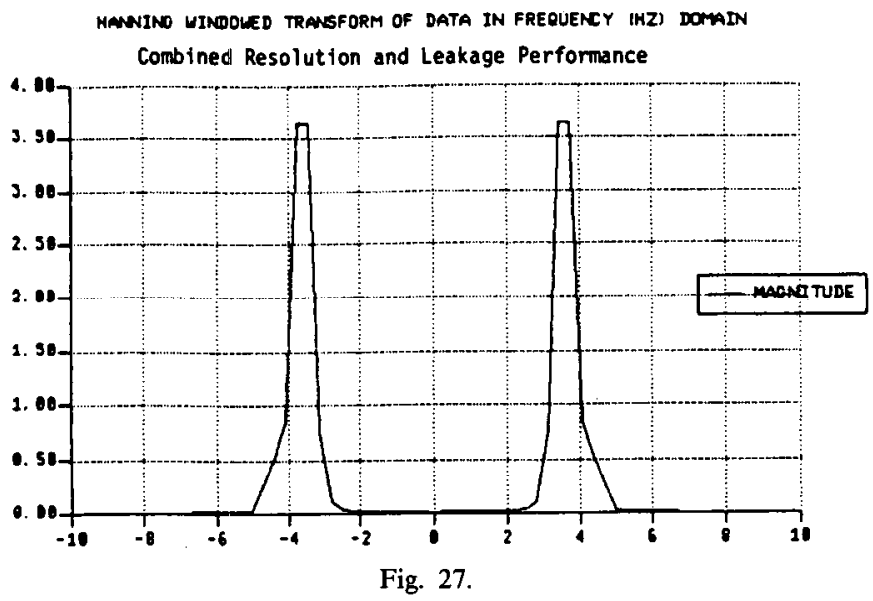

well as the rectangular window in this respect, while the Hanning and Hamming windows both fail to distinguish the two frequencies.

The third comparison among the windows combines the considerations of leakage and resolvability. The windows were applied to a data sequence comprised of an amplitude 10 component at $11.5 \Delta_{f}^{(1)}=3.59375 \mathrm{~Hz}$, and another sinusoid of amplitude 1.25 at $14 \Delta_{f}^{(1)}=4.375 \mathrm{~Hz}$. The results of this test appear in Figs. 25-28 (generated, rectangular, Hanning and Hamming, respectively). It is apparent that the window generated by the iterative synthesis technique is the only one of the four to clearly distinguish the presence of a discrete lower amplitude frequency compo-

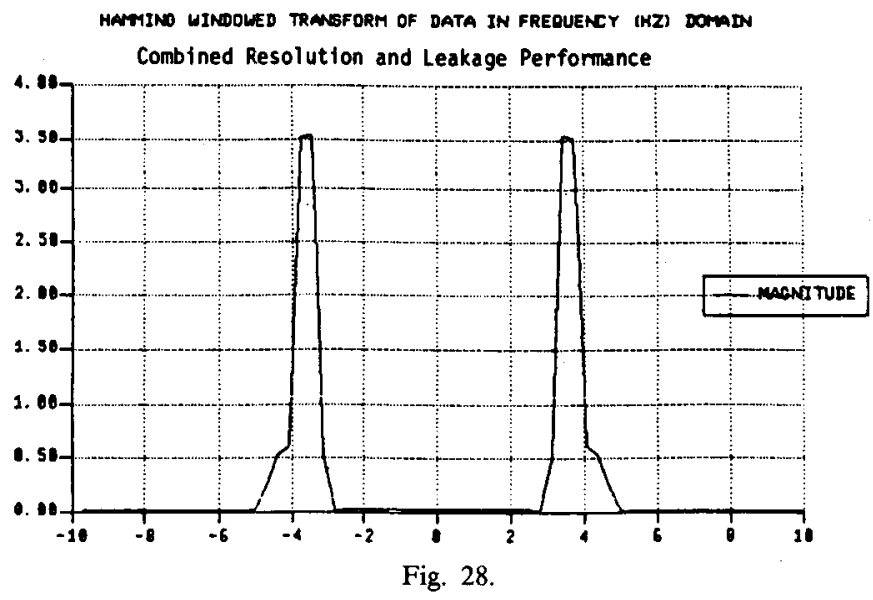

nent. Of the remaining three, the performance of the Hamming window is the most appealing, as would be expected.

It is hoped that this example has demonstrated the utility of the synthesis technique of this paper. By satisfying a constraint of finite time duration, while nearly satisfying a constraint upon the spectral content of the signal, a data window was generated whose ability to resolve proximal frequencies of greatly varying amplitudes is superior to that of three commonly employed windows.

\section{REMARKS}

In the course of any research endeavor, the treatment of certain germane topics will be precluded by limitations upon the available time and space. The intent of Section IV is to remark upon some of those topics and to mention aspects of the synthesis technique of this paper which may benefit from further research.

The reader may recall that Theorem 3 of Section II established that for each $\left(x_{1}, x_{2}\right) \in D_{P},\left\|x_{1}-x_{2}\right\|=$ $d\left(C_{1}, C_{2}\right)$-with the assumption that the underlying Hilbert Space was defined over the scalar field of real numbers. It would clearly be desirable to extend the Theorem to Hilbert Spaces which are defined over the scalar field of complex numbers and, hence, to make possible the synthesis of complex-valued functions within the context of a single space. In its present form, Theorem 3 necessitates a product space structure, of the type mentioned in the discussion preceding that theorem, in order that it be applicable to complex-valued functions. To effect such an extension, it will be, in the authors' opinion, necessary to change the manner in which the Theorem is proven. The current proof relies, to a significant extent, upon the sign of the inner product of certain elements in the space-a notion which becomes tenuous when the inner product is allowed to assume complex values. Theorem 3 is the only result of Section II which is dependent upon the scalar field over which the underlying space is defined.

It is the authors' belief that the utility of the synthesis technique of this paper will be conditional upon the construction of a more extensive collection, than that which is currently available, of convex sets corresponding to meaningful signal constraints, and of their corresponding nearest point maps. Although the presentation of this 
paper was phrased in terms of signal synthesis, the technique may find application in the more general class of constrained optimization problems, in which the severity of the various constraints generates an inconsistency. Some examples might include the design of control systems and the design of manufacturing processes. As in the case of signal synthesis, however, the identification of convex sets corresponding to meaningful design constraints, and of their associated nearest point maps, will be a prerequisite.

The discussions in this paper were confined to the case of two, disjoint, closed convex sets. Consider the case of $N$, mutually disjoint, closed convex sets $\left\{C_{1}, \cdots, C_{N}\right\}$, with respective projection operators $P_{1}, \cdots, P_{N}$. Suppose that $C_{1}$ is bounded, and form the composite operator $P=$ $P_{1} \circ \cdots \circ P_{N}$. Then, by Theorems 2 and 4 of Section II, respectively, the set of fixed points of $P$ is nonempty and $P_{\lambda}=\lambda I+(1-\lambda) P, 0<\lambda<1$, is asymptotically regular. Furthermore, by Theorem 5, the sequence $\left\{\boldsymbol{P}_{\lambda}^{n}(P x)\right\}_{n=1}^{\infty}$ is weakly convergent to a fixed point of $P$ for an arbitrary element $x$ of the underlying Hilbert Space. Thus the formal mathematical extension of the technique of this paper, to any finite number of mutually disjoint, closed convex sets, is feasible. The questions which must be resolved, in this case, are those of a physical interpretation for the results of an application of the technique. Specifically, the relationship of the fixed points of $P$ to the elements of the remaining $N-1$ sets must be ascertained. In the case of two disjoint sets, if $x_{1}$ was an element of the set of fixed points of $P=P_{1} \circ P_{2}$, it was demonstrated that $\left\|x_{1}-P_{2} x_{1}\right\|$ was minimized. In the general case, preliminary results have shown that

$$
\begin{aligned}
&\left\|x_{1}-P_{N} x_{1}\right\|+\left\|P_{N} x_{1}-\left(P_{N-1} \circ P_{N}\right) x_{1}\right\|+\cdots \\
&+\left\|\left(P_{2} \circ \cdots \circ P_{N}\right) x_{1}-\left(P_{1} \circ \ldots \circ P_{N}\right) x_{1}\right\|
\end{aligned}
$$

where $x_{1}$ is a fixed point of $P=P_{1} \circ \ldots \circ P_{N}$, is not necessarily equal to the minimal length of a closed polygonal path with a single vertex in each set.

\section{ACKNOWLEDGMENT}

The authors are indebted to Prof. Victor Klee, of the Mathematics Department at the University of Washington, Seattle, for suggesting the scheme of the proof for Theorem 3 of Section II. They are also grateful for the assistance provided by Prof. Mark Damborg, of the University of Washington's Electrical Engineering Department, in the formulation of the results of Section II.

\section{REFERENCES}

[1] Ruel V. Churchill, J. Brown, and R. Verhey, Complex Variables and Applications. New York: McGraw-Hill, 1976.

[2] J. R. Fienup, "Reconstruction and Synthesis Applications of an Iterative Algorithm," Transformations in Optical Signal Processing, Proc. SPIE, V. 373 1981, pp. 147-160.

[3] A. Papoulis, "A New Method of Image Restoration", Joint Services Technical Advisory Committee Rep. 39, 1973-74.

[4] "A new algorithm in spectral analysis and band-limited extrapolation," IEEE Trans. Circuits Syst., vol. CAS-22, pp. 735-742, 1975.

[5] R. W. Gerchberg, "Super-resolution through error energy reduction," Opt. Acta, vol. 21, pp. 709-720, 1974.

[6] Dante C. Youla, "Generalized image restoration by the method of alternating orthogonal projections," IEEE Trans. Circuits Syst., vol. CAS-25, pp. 694-702, 1978.
[7] H. Stark, D. Cahana, and H. Webb, "Restoration of arbitrary finite-energy optical objects from limited spatial and spectral information," J. Opt. Soc. Amer., vol. 71, pp. 635-642, 1981.

[8] J. A. Cadzow, "An extrapolation procedure for band-limited signals," IEEE Trans. Acoust. Speech, Signal Processing, vol. ASSP-27, pp. 4-12, 1979.

[9] R. J. Marks, II, and D. K. Smith, "Gerchberg-type linear deconvolution and extrapolation algorithms," Transformations in Optical Signal Processing, Proc. SPIE, vol. 373, pp. 161-178, 1981.

[10] Dante C. Youla, and H. Webb, "Image restoration by the method of convex projections: Part 1 -Theory," IEEE Trans. Med. Imaging, vol. MI-1, pp. 81-94, 1982.

[11] James A. Clarkson, "Uniformly convex spaces," Trans. Amer. Math. Soc., vol. 40 (1936), pp. 396-414.

[12] Kosaku Yosida, Functional Analysis. New York, Springer-Verlag, Fifth ed., 1978 .

[13] Helmut Schaefer, "Uber Die Methode Sukzessiver Approximationen," Jahresbericht Deutsche Math. Vereinigung, vol. 59, pp. 131-140, 1957.

[14] L. G. Gubin, B. Polyak, and E. Raik, "The method of projections for finding the common point of convex sets," USSR J. of Comput. Math. Math. Phys., vol. 7, \#6, pp. 1211-1228, 1967.

[15] R. R. Phelps, "Convex sets and nearest points," Proc. Amer. Math. Soc., vol. 8, pp. 790-797, 1957.

[16] _ , "Convex sets and nearest points, II," Proc. Amer. Math. Soc., vol. 9, pp. 867-873, 1958.

[17] Felix E. Browder, "Nonexpansive nonlinear operators in a Banach Space," in Proc. Nat. Acad. Sciences, vol. 54, pp. 1041-1044, 1965.

[18] Zdzislaw Opial, "Weak convergence of the sequence of successive approximations for nonexpansive mappings," Bull. Amer. Math. Soc., vol. 73, pp. 591-597, 1967.

[19] David G. Luenberger, Optimization by Vector Space Methods. New York: Wiley, 1969.

[20] Felix E. Browder and W. Petryshyn, "The solution by iteration of nonlinear functional equations in Banach Spaces," Bull. Amer. Math. Soc., vol. 72, pp. 571-575, 1966

[21] Ronald W. Schafer, R. Mersereau, and M. Richards, "Constrained iterative restoration algorithms," Proc. IEEE, vol. 69, pp. 432-450, 1981.

[22] Steven Kay and S. Marple, "Spectrum analysis-A modern perspective," Proc. IEEE, vol. 69, pp. 1380-1419, 1981.

[23] Frederic Harris, "On the use of windows for harmonic analysis with the discrete Fourier transform," Proc. IEEE, vol. 66, pp. 51-83, 1978.

[24] Alan V. Oppenheim and R. Schaefer, Digital Signal Processing. Englewood Cliffs: NJ: Prentice-Hall, 1975.

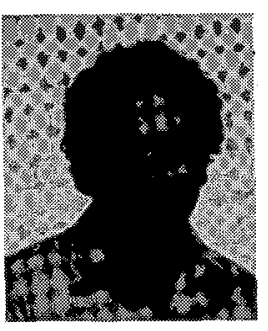

Marc Goldburg (S'83-M'84) was born on October 24,1959 . He received the B.S.E. degree in electrical engineering from Princeton University, Princeton, NJ, in 1982, and the M.S.E.E. degree from the University of Washington, Seattle, WA, in 1984 .

His research interests include multidimensional signal processing; signal synthesis and reconstruction; stochastic systems; nonuniform sampling schemes; and mathematical applications. He is currently a staff member of the surviellance systems group at Massachusetts Insititute of Technology's Lincoln Laboratory, where he is participating in the development of signal processing and automated forecasting techniques for weather radar systems.

Mr. Goldburg is a member of SIAM and Sigma Xi.

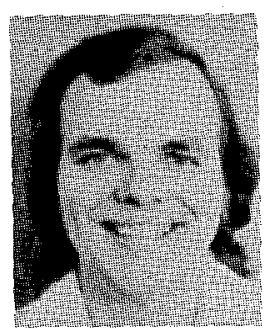

Robert J. Marks II (S'76-M'77-SM'83) was born in Sutton, WV , on August 25, 1950. He received the B.S. and M.S. degrees in electrical engineering from the Rose-Hulman Institute of Technology, Terre Haute, IN, in 1972 and 1973, respectively, and the Ph.D. degree from Texas Tech University, Lubbock, in 1977

From 1973 to 1975 , he served as a Reliability Engineer with the U.S. Department of the Navy. Presently, he is an Associate Professor of Elcctrical Engineering at the University of Washington, Seattle. Areas of interest include signal processing, statistical communication theory, and optical processing. He has published over forty refereed journal and proceedings papers in these areas.

Dr. Marks is a member of OSA, SPIE, Eta Kappa Nu, and Sigma XI In 1982, he was awarded the Outstanding IEEE Branch Counciler Award by IEEE and in 1984 was a recipient of the IEEE Centennial Medal and Certificate. 